\title{
GEOPHYSICS
}

\section{A least-squares correlation-based full traveltime inversion for shallow subsurface velocity reconstruction}

\begin{tabular}{|r|l|}
\hline Journal: & Geophysics \\
\hline Manuscript ID & GEO-2018-0581.R2 \\
\hline Manuscript Type: & Technical Paper \\
\hline Keywords: & $\begin{array}{l}\text { correlation, full-waveform inversion, near surface, traveltime, wave } \\
\text { propagation }\end{array}$ \\
\hline Area of Expertise: & Seismic Inversion, Seismic Modeling and Wave Propagation \\
\hline \multicolumn{2}{|l}{} \\
\end{tabular}

\section{SCHOLARONE \\ Manuscripts}




\section{A least-squares correlation-based full traveltime inversion for shallow subsurface velocity reconstruction}

Jia $\mathrm{Yi}^{1,2}$, Yike Liu ${ }^{1}$, Zongqi Yang ${ }^{3}$, Huiyi $\mathrm{Lu}^{1}$, Bin $\mathrm{He}^{1}$, and Zhendong Zhang ${ }^{4}$

Right Running Head: LCFTI for velocity reconstruction

${ }^{1}$ Key Laboratory of Petroleum Resource Research, Institute of Geology and Geophysics,

Chinese Academy of Sciences, Beijing, China. E-mail: yijia@mail.iggcas.ac.cn;

ykliu@mail.iggcas.ac.cn; lhy061981@163.com; hebin@mail.iggcas.ac.cn.

${ }^{2}$ University of Chinese Academy of Sciences, Beijing, China. E-mail:

yijia@mail.iggcas.ac.cn.

${ }^{3}$ BGP INTERNATIONAL, CNPC, Hebei, Zhuozhou, China. E-mail:

yangzongqi@bgpintl.com.

${ }^{4}$ King Abdullah University of Science and Technology, Thuwal, Saudi Arabia. E-mail:

Zhendong.zhang@kaust.edu.sa. 


\section{ABSTRACT}

The accurate estimation of shallow subsurface velocity models with complex topography is crucial for statics corrections and imaging deep structures. The correlation-based wave-equation traveltime inversion (CWTI) method is suitable for estimating such shallow subsurface velocity structures. However, the CWTI objective function suffers an inherent resolution-loss problem since the traveltime weighted crosscorrelation misfit does not fall to zero even when the model is perfectly matched. Furthermore, the Born-approximation-based CWTI gradient cannot provide an effective model update during each iteration. To overcome these problems, we propose a least-squares correlation-based full traveltime inversion (LCFTI) method in which the least-squares correlation-based objective function is designed to minimize the traveltime weighted difference between the autocorrelation and the crosscorrelation. By incorporating the autocorrelation, LCFTI shows better convergence and higher resolution than CWTI. The LCFTI model updates are derived using the Rytov approximation to avoid incorrect model updates by emphasizing phase matching. Furthermore, to accurately simulate wave propagation, we use the spectral-element method as the modeling engine in which the mesh of the complex topography is flexibly represented. Synthetic and field data examples are shown to demonstrate the effectiveness of the proposed method in shallow subsurface velocity reconstruction. 


\section{INTRODUCTION}

The complexity of shallow subsurface structures leads to complex scattering. Accurately simulating seismic wave propagation in the presence of curved topography is essential for complex shallow subsurface inversion and imaging. The commonly used finite-difference method can handle smoothly varying topography if it is implemented on a curved mesh that honors the shape of the topography (Zhang and Chen, 2006; Tarrass et al., 2011; Pérez Solano et al., 2016). However, it is hard to adapt to complex topography. In recent years, the spectral-element method (SEM) has been introduced in exploration seismology as a powerful modeling tool (Komatitsch and Vilotte, 1998; Liu et al., 2014) combining the geometrical flexibility of the low-order finite-element method with the accuracy associated with the spectral method. Consequently, SEM has been applied in many studies due to its high accuracy and flexible meshing of complex topography.

Besides the accurate seismic wavefields, the resolution of the shallow subsurface velocity structure is another significant factor affecting high-precision imaging. First-arrival traveltime tomography (Lanz et al., 1998; Martí et al., 2008; Adamczyk et al., 2013) has been extensively used in industry to recover the velocity of shallow subsurface P-waves due to its high-efficiency and robustness. However, traveltime tomography relies on the high-frequency approximation, which is not valid for complex media. On the other hand, surface-wave dispersion curve analysis (Sacchi and Ulrych, 1995; Park et al., 1999; Zhang et al., 2016) is a powerful tool for 1D shear-wave velocity estimation for shallow depths. The 1D profiles can be 
inverted jointly to reconstruct a pseudo-2D velocity structure (Boiero and Socco, 2010; Bergamo et al., 2012; Mi et al., 2017). However, because this method does not directly consider the effect of $2 \mathrm{D}$ inhomogeneities in the medium, the estimates tend to have low horizontal resolution.

Full-waveform inversion (FWI) has been developed for inverting for subsurface structures (P- and S-wave velocity, density, and other parameters) from the observed signals by utilizing all information contained in the waveforms in the inversion strategy (Virieux and Operto, 2009). Therefore, FWI has shown great potential for yielding high-resolution estimates even for complex media. Early-arrival waveform inversion (Sheng et al., 2006; Shen, 2010; Adamczyk et al., 2014) has been widely used to estimate shallow subsurface velocity structures as it can provide supplemental and short-wavelength information for shallow seismic data. Surface-wave FWI-based approaches (Pérez Solano et al., 2014; Yuan et al., 2015; Masoni et al., 2016) have been shown to handle lateral variation by taking advantage of the information contained in surface waves to image heterogeneous shallow structures. However, all conventional FWI methods suffer from severe cycle-skipping problem when the starting model is far from the true model or if sufficiently low frequencies are not available in the seismic data (Zhou et al., 2012). Thus, these conventional L2-norm-based FWI approaches can be highly nonlinear and tend to convergence at local minima.

As a result, numerous alternative methods have been proposed for resolving these issues. A multiscale strategy has been designed to mitigate the nonlinearity by 
utilizing frequency ranges from low to high (Bunks et al., 1995). Laplace-domain and Laplace-Fourier-domain FWI approaches have been proposed to reduce the local minima, both of which are multiscale approaches in the data domain (Shin and Cha, 2008, 2009). Almomin and Biondi (2012) proposed a tomographic FWI approach that focuses on long-wavelength component recovery. Adaptive waveform inversion (Warner and Guasch, 2016) uses a Wiener filter to achieve adaptive data matching and shows immunity to cycle-skipping. Many other methods including envelope inversion (Wu et al., 2014), direct waveform inversion (Liu and Zheng, 2015), unwrapped phase inversion (Choi and Alkhalifah, 2015), the selective data extension approach (Wu and Alkhalifah, 2018), and full-intensity waveform inversion (Liu et al., 2018) have been able to mitigate the cycle-skipping problem.

The amplitude and traveltime (phase) have different sensitivities to inversion, and FWI is more likely to fail in the presence of amplitude errors. Therefore, many researchers prefer the wave-equation traveltime inversion scheme (Luo and Schuster, 1991; de Hoop and van der Hilst, 2005; Zhang and Wang, 2009; Ma and Hale, 2013; Zhang et al., 2015), which emphasizes traveltime matching and is less sensitive to amplitude errors in seismic data. Wave-equation traveltime inversion has a more linear relationship between its objective and the model making it more robust than waveform inversion. In particular, the classic wave-equation traveltime inversion method proposed by Luo and Schuster (1991) is a very robust and kinematic approach. However, this method is very sensitive to errors in the estimated source signature (de Hoop and van der Hilst, 2005) because its crucial step, which relies on 
picking the correlation maximum, will fail when the source spectra are not identical. Therefore, van Leeuwen and Mulder (2010) introduced a widely used correlation-based wave-equation traveltime inversion (CWTI) (Fichtner, 2011; Luo and Sava, 2011; Luo et al., 2016), which implicitly measures the traveltime shifts and is less sensitive to errors of source spectra. Moreover, minimizing the norm of the traveltime weighted crosscorrelation as an optimization criterion is less susceptible to the cycle-skipping problem. Because most of the energy in the correlation is contributed by early arrivals, CWTI might be appropriate for the inversion of the shallow subsurface structure. However, Choi and Alkhalifah (2016) observed that the correlation-based misfit does not go to zero when the inverted model is the exact model, and this limitation can be overcome by incorporating the autocorrelation of the observed data in their optimized correlation-based objective function. Yi and Liu (2017) showed that the gradient of the CWTI, which is derived based on the Born approximation (Clayton and Stolt, 1981), cannot provide an effective model update during the iterations. In particular, the Born-approximation-based gradient cannot separate the effects of traveltime and amplitude, which is not consistent with the traveltime-matching targeted-fitting process. Hence, Luo et al. (2016) proposed a full traveltime inversion (FTI) approach based on the Rytov approximation (Rajan and Frisk, 1989) in which velocity perturbations are assumed only to cause traveltime changes and the influence of amplitude in the inversion is ignored.

In this study, we propose a least-squares correlation-based full traveltime inversion (LCFTI) approach for complex shallow subsurface velocity reconstruction. 


\section{CONVENTIONAL CORRELATION-BASED WAVE-EQUATION TRAVELTIME INVERSION}

An important procedure in wave-equation traveltime inversion approaches involves calculating the traveltime differences (or relative phase shifts) between the calculated and observed data. The CWTI approach (van Leeuwen and Mulder, 2010) was proposed to implicitly measure these traveltime differences as an optimization criterion. The correlation-based objective function of the CWTI approach can be written as

$$
M_{C W T I}=\frac{1}{2} \sum_{\boldsymbol{r}_{g}, \boldsymbol{r}_{s}} \int\left[W(\tau) \cdot C_{\text {cross }}\left(\boldsymbol{r}_{g}, \tau ; \boldsymbol{r}_{s}\right)\right]^{2} d \tau
$$


where $\boldsymbol{r}_{g}$ and $\boldsymbol{r}_{s}$ indicate the receivers and sources location, respectively. Moreover, $C_{\text {cross }}\left(\boldsymbol{r}_{g}, \tau ; \boldsymbol{r}_{s}\right)$ describes the crosscorrelation between the observed data $p_{o}$ and calculated data $p_{c}$ with traveltime shift $\tau$ as

$$
C_{\text {cross }}\left(\boldsymbol{r}_{g}, \tau ; \boldsymbol{r}_{s}\right)=\int p_{c}\left(\boldsymbol{r}_{g}, t ; \boldsymbol{r}_{s}\right) p_{o}\left(\boldsymbol{r}_{g}, t+\tau ; \boldsymbol{r}_{s}\right) d t
$$

Figure 1 illustrates the correlation process using two traces. $W(\tau)$ is a weighting function used to penalize energy at non-zero time shifts. In particular, $W(\tau)$ can be a linear function within the selected time window (Shen and Symes, 2008; van Leeuwen and Mulder, 2010; Zhang et al., 2018)

$$
W(\tau)= \begin{cases}\tau & \text { if }|\tau| \leq \tau_{\max } \\ 0 & \text { otherwise }\end{cases}
$$

where $\tau_{\max }$ is the maximum traveltime shift. The gradient and adjoint source corresponding to the objective function are provided in Appendix A.

Though the CWTI scheme is suitable for estimating shallow subsurface velocity structure and is resistant to the cycle-skipping problem, CWTI is prone to severe resolution loss when the inverted model is close to the actual model. Here, we use a Gaussian-circle inclusion velocity model to demonstrate the resolution-loss problem. The true model is shown in Figure 2a, where the source used for generating the data is a band-limited Ricker wavelet with a peak frequency of $50 \mathrm{~Hz}$. In the true model, 201 point sources are evenly distributed on the left side with 201 receivers on the right side. Figure 3a shows common-shot gathers calculated with the true velocity. We use the true model as a trial model to calculate the corresponding gradient and adjoint source for the CWTI algorithm, as shown in Figures $2 \mathrm{~b}$ and $3 \mathrm{~b}$. The adjoint source is nonzero, which leads to a nonzero gradient (Figure 2b) and an error velocity 
update, even though the model is correct. The correlation-based objective function does not reduce to zero when the model misfit becomes zero. Due to the energy of crosscorrelation, which cannot be annihilated by the weighting function for the band-limited wavelet, the objective function can only be zero when the pulse signal is used.

\section{LEAST-SQUARES CORRELATION-BASED FULL TRAVELTIME INVERSION}

To mitigate the resolution-loss problem in the conventional correlation-based objective function, we define the autocorrelation $C_{\text {auto }}$ of the observed data as

$$
C_{\text {auto }}\left(\boldsymbol{r}_{g}, \tau ; \boldsymbol{r}_{s}\right)=\int p_{o}\left(\boldsymbol{r}_{g}, t ; \boldsymbol{r}_{s}\right) p_{o}\left(\boldsymbol{r}_{g}, t+\tau ; \boldsymbol{r}_{s}\right) d t .
$$

Note that the energy of autocorrelation will reach its maximum when the traveltime shift reduces to zero, as shown in Figure 1b, which coincides with minimization of the conventional correlation-based objective function. Therefore, we propose a least-squares correlation-based objective function:

$$
M_{L C F T I}=\frac{1}{2} \sum_{\boldsymbol{r}_{g}, r_{s}} \int\left\{W(\tau)\left[C_{\text {auto }}\left(\boldsymbol{r}_{g}, \tau ; \boldsymbol{r}_{S}\right)-C_{\text {cross }}\left(\boldsymbol{r}_{g}, \tau ; \boldsymbol{r}_{S}\right)\right]\right\}^{2} d \tau
$$

The autocorrelation acts as a reference value in the inversion similar to the observed data in conventional FWI. Unlike FWI, the function $W(\tau)$ in equation 3 is introduced to penalize the subtraction correlation result at nonzero traveltime shift. Under this circumstance, if the starting model is far from the exact model, the inversion emphasizes traveltime matching. When the inverted model is close to the exact model, $W(\tau)$ suppresses its impact, enabling the inversion to focus on 
matching the amplitude; meanwhile, the energy of the subtraction correlation will reduce to zero. As a result, the cycle-skipping problem faced by conventional FWI can be mitigated, and the resolution-loss problem in the conventional correlation-based objective function can also be avoided.

To derive the corresponding gradient and adjoint source, we use the Rytov approximation instead of the widely used Born approximation. The Rytov approximation is derived by assuming the wavefield is a plane wave defined as $p(\omega)$ $=A e^{\Psi}=A e^{i \omega t}$, where $A, \Psi$, and $t$ indicate the amplitude, complex wavefield phase, and traveltime, respectively. Note that in the Rytov wavepath, phase and amplitude separate naturally (Chi et al., 2012; Luo et al., 2016). However, phase and amplitude cannot be separated in the Born-approximation wavepath. Therefore, if the effect of amplitude is to be deemphasized, the Rytov approximation is more suitable for solving wave-equation traveltime inverse problems. Assuming the background wavefield is given as $p_{0}(\omega)=A_{0} e^{\Psi_{0}}=A e^{i \omega t_{0}}$, where $A_{0}, \Psi_{0}$, and $t_{0}$ are the related amplitude, complex wavefield phase, and traveltime, respectively, the complex wavefield-phase perturbation $\Delta \Psi(\omega)$ based on the Rytov approximation can be written as

$$
\Delta \Psi(\omega)=\ln (p(\omega))-\ln \left(p_{0}(\omega)\right)=\ln \left(\frac{A}{A_{0}}\right)+i \omega \Delta \tau,
$$

where $\Delta \tau=t-t_{0}$ is the traveltime shift. Equation 6 indicates that traveltime and amplitude are separated into real and imaginary parts. The amplitude is subjected to a variety of effects that may cause highly nonlinear behavior during wave-equation 
traveltime inversion. Therefore, by removing the amplitude-related term $\ln \left(\frac{A}{A_{0}}\right)$ in equation 6 , we assume that the model perturbation $\Delta s$ only causes traveltime changes $\Delta \tau$ in the wavefield (Luo et al., 2016):

$$
\left\{\begin{array}{c}
p_{c}\left(\boldsymbol{r}_{g}, t ; \boldsymbol{r}_{s}\right)_{s_{0}+\Delta s}=p_{c}\left(\boldsymbol{r}_{g}, t+\Delta \tau ; \boldsymbol{r}_{s}\right)_{s_{0}} \\
p_{c}\left(\boldsymbol{r}_{g}, t ; \boldsymbol{r}_{s}\right)_{s_{0}}=p_{o}\left(\boldsymbol{r}_{g}, t+\Delta \tau ; \boldsymbol{r}_{s}\right)_{s_{0}}
\end{array},\right.
$$

where $s_{0}$ is the background slowness, and $p_{c}$ and $p_{o}$ are the calculated and observed data, respectively.

Then, the gradient $\chi_{\boldsymbol{R}}$ corresponding to the proposed LCFTI with respect to the slowness model $s$ for any location $\boldsymbol{r}^{\prime}$, which is based on the Rytov approximation, can be written as

$$
\chi_{\boldsymbol{R}}=\frac{\partial M_{L C F T I}}{\partial s\left(\boldsymbol{r}^{\prime}\right)}=\sum_{\boldsymbol{r}_{g}, \boldsymbol{r}_{s}} \int W(\tau)^{2}\left(C_{\text {auto }}-C_{\text {cross }}\right)\left[-\frac{\partial C_{\text {cross }}}{\partial \tau} \frac{\partial \Delta \tau}{\partial s\left(\boldsymbol{r}^{\prime}\right)}\right] d \tau
$$

Here, we utilize the chain rule $\left[\frac{\partial C}{\partial(t+\Delta \tau)}\right]\left[\frac{\partial(t+\Delta \tau)}{\partial s\left(\boldsymbol{r}^{\prime}\right)}\right]=\left[\frac{\partial C}{\partial \tau}\right]\left[\frac{\partial \Delta \tau}{\partial s\left(\boldsymbol{r}^{\prime}\right)}\right]$ given in appendix A of Luo et al. (2016). The Fréchet derivative $\frac{\partial \Delta \tau}{\partial s\left(\boldsymbol{r}^{\prime}\right)}$ with respect to the slowness $s\left(\boldsymbol{r}^{\prime}\right)$ is given by

$$
\frac{\partial \Delta \tau}{\partial s\left(\boldsymbol{r}^{\prime}\right)}=-2 s_{0}\left(\boldsymbol{r}^{\prime}\right) \frac{\int \dot{p}_{o}\left(\boldsymbol{r}_{g}, t+\tau ; \boldsymbol{r}_{s}\right) G\left(\boldsymbol{r}^{\prime}, t ; \boldsymbol{r}_{g}\right) * \ddot{p}_{c}\left(\boldsymbol{r}^{\prime}, t ; \boldsymbol{r}_{s}\right) d t}{\int \dot{p}_{o}\left(\boldsymbol{r}_{g}, t+\tau ; \boldsymbol{r}_{s}\right) \dot{p}_{o}\left(\boldsymbol{r}_{g}, t+\tau ; \boldsymbol{r}_{s}\right) d t}
$$

where $G$ is the Green's function, the notation $*$ denotes time convolution, and $\dot{p}_{o}$ and $\ddot{p}_{c}$ represent the first and second time derivative of the wavefield, respectively. The detailed derivation of equation 9 can be found in Appendix B. Substituting equation 9 into equation 8, the LCFTI gradient is represented as

$$
\chi_{\boldsymbol{R}}=-4 s_{0}\left(\boldsymbol{r}^{\prime}\right) \sum_{\boldsymbol{r}_{g}, \boldsymbol{r}_{s}} \int \ddot{p}_{c}\left(\boldsymbol{r}^{\prime}, t ; \boldsymbol{r}_{s}\right) \cdot P_{R}^{\prime} d t
$$


where the adjoint wavefield $P_{R}^{\prime}$ is generated by back-propagating the following adjoint source:

$$
\boldsymbol{\delta}_{\boldsymbol{R}}=\int W(\tau) \frac{\left(C_{\text {auto }}-C_{\text {cross }}\right) \cdot C_{\text {cross }} \cdot \dot{p}_{o}\left(\boldsymbol{r}_{g}, t+\tau ; \boldsymbol{r}_{s}\right)}{\int \dot{p}_{o}\left(\boldsymbol{r}_{g}, t+\tau ; \boldsymbol{r}_{s}\right) \dot{p}_{o}\left(\boldsymbol{r}_{g}, t+\tau ; \boldsymbol{r}_{s}\right) d t} d \tau
$$

A detailed derivation of the LCFTI gradient and the adjoint source can be found in Appendix C.

\section{Behaviors of the least-squares correlation-based objective function}

To examine the behavior of the proposed least-squares correlation-based objective function, we first compare it with the waveform-subtraction objective function of traditional FWI. A simple homogeneous velocity model is used to investigate their sensitivities to cycle-skipping. As shown in Figure 4a, within the range of given velocity errors, the waveform-subtraction objective function of the traditional FWI has more than one local minimum, whereas our proposed objective function always has a global minimum, suggesting it is less sensitive to velocity errors. In addition, the proposed objective function has a large basin of attraction of the global minimum, therefore, it is well suited for low-wavenumber background velocity reconstruction.

The proposed objective function is also compared with the conventional correlation-based objective function of CWTI. Here, we use the Gaussian-circle inclusion model (Figure 2a) as the true model, and the trial model is built by perturbing the Gaussian anomaly. Figure $4 \mathrm{~b}$ shows that the proposed least-squares correlation-based objective function drops to zero when the trial model is exactly the 
same as the actual model, whereas the conventional correlation-based objective function still shows a misfit residual. This comparison proves that an inherent resolution-loss problem exists in the conventional correlation-based objective function and that the proposed objective function curve is more convex, improving its convergence.

\section{Effectiveness of the Rytov-approximation-based LCFTI gradient}

To investigate the effectiveness of the Rytov-approximation-based LCFTI gradient, we use the homogeneous model with one source and receiver pair in a transmission acquisition setting together with two initial models having relatively lower and relatively higher velocities. The sensitivity kernels of LCFTI with respect to the initial models are shown in Figures $5 \mathrm{a}$ and $5 \mathrm{~b}$. Figure $5 \mathrm{a}$ indicates a positive gradient direction in the first Fresnel zone, which plays a major role in the model update criterion for the lower velocity initial model, and Figure 5 b provides a negative gradient direction for the higher velocity initial model. The results demonstrate the correct gradient directions with the Rytov-approximation-based LCFTI gradient. For a better comparison, we also provide the sensitivity kernels of the Born-approximation-based CWTI in Figures 5c and 5d, which both show the same positive gradient direction. The gradient in Figure $5 \mathrm{c}$ is in the correct direction, while the gradient in Figure 5d fails to distinguish that the initial velocity model is higher than the true model.

We further test the LCFTI gradient with the Gaussian-circle inclusion model 
(Figure 2a). Two starting velocity models, which have smaller or larger Gaussian perturbations, are used to calculate the corresponding LCFTI gradients, and the background velocity is the same as the true model. The gradients are represented in Figure $6 \mathrm{a}$ and $6 \mathrm{~b}$. These results further prove that the LCFTI gradient could effectively indicate the correct model update direction. The CWTI gradients shown in Figures $6 \mathrm{c}$ and $6 \mathrm{~d}$ demonstrate that the CWTI gradient fails to provide the correct direction in the case of the larger Gaussian perturbation.

The failure of the CWTI gradient in Figure $5 \mathrm{~d}$ and Figure $6 \mathrm{~d}$ can be explained as follows. When a relatively higher or larger velocity model results in a negative traveltime difference $(-\tau)$, the negative traveltime difference is squared $\left(\mathrm{W}(\tau)^{2}\right)$ in the CWTI adjoint source (equation $\mathrm{A}-3$ ), thus producing the same gradient direction as the relatively lower or smaller initial model, which has a positive traveltime difference. However, because there is only a single weighting function in the LCFTI adjoint source (equation $\mathrm{C}-6$ ) used in the calculation of the gradient (equation C -2 ), the LCFTI gradients always generate the correct direction.

\section{EXAMPLES}

In this section, we demonstrate the performance and advantages of our proposed method using two synthetic examples and one field data set. The first synthetic example is an experiment on a crosswell data set intending to verify the effectiveness of the proposed LCFTI. In the second example, we utilize the Canadian foothill model to analyze the performance of our method for complex shallow 
subsurface velocity reconstruction. Finally, LCFTI is applied to a shallow marine data set to test the applicability of the method in field data processing.

\section{Crosswell model}

We first apply LCFTI to a crosswell model, as shown in Figure 7a. The model contains 132 and 329 grid points in the $\mathrm{x}$ - and z-direction, respectively, with a constant sampling of $1 \mathrm{~m}$, and 110 sources and 110 receivers are distributed vertically along the sides of the model at a constant distance of $3 \mathrm{~m}$. We generate the observed data using a Ricker-wavelet source with a peak frequency of $300 \mathrm{~Hz}$. The starting velocity model is a homogeneous model far from the actual model, as shown in Figure $7 \mathrm{~b}$, in which case the conventional FWI would fail. Figures $7 \mathrm{c}$ and $7 \mathrm{~d}$ show the inverted results using CWTI and our proposed LCFTI. The LCFTI approach is effective since it recovers a good low-wavenumber background velocity model (Figure 7d), even with a poor starting model, while the CWTI fails to do so. We then use the LCFTI result (Figure 7d) as an initial model for the conventional FWI to obtain the high-resolution velocity model shown in Figure 7e, which indicates that LCFTI could provide a good model for conventional FWI.

The residual of the objective function with respect to the iterations for LCFTI and CWTI is shown in Figure 8. In this experiment, LCFTI converges much faster than CWTI. Although the objective function of CWTI declines, the updates are in the wrong direction, so the objective function drops by less than $40 \%$. 


\section{Canadian foothill model}

Next, we test the proposed method on the Canadian foothill model (Gray and Marfurt, 1995), which consists of a rugged surface and many complicated structures in the shallow depth. The model has $833 \times 200$ grid points with a spacing of $10 \mathrm{~m}$, as shown in Figure 9a. There are 103 sources and 833 receivers distributed evenly on the rugged surface with samplings of $80 \mathrm{~m}$ and $10 \mathrm{~m}$, respectively. The maximum offset is $4000 \mathrm{~m}$. We generate the observed data using SPECFEN2D, a 2D SEM code (Komatitsch and Vilotte, 1998), with a Ricker-wavelet of peak frequency of $10 \mathrm{~Hz}$. Figure 9c illustrates the irregular quadrilateral mesh used by SEM.

To approximate the field data condition, we remove frequencies below $5 \mathrm{~Hz}$, as shown in Figure 10a, and we start from the homogeneous velocity model shown in Figure $9 b$, which is far from the true model. Figure $10 \mathrm{~b}$ shows the comparison of traces between the observed and calculated data based on the actual and initial model. Note that the differences between the observed and calculated data are out of phase by more than a half cycle in the long offset, which is sufficient to introduce cycle-skipping into conventional FWI. For comparison, we conduct a simple multiscale FWI (Bunks et al., 1995) and our proposed LCFTI using the same parameters. Four different scales $(5-8 \mathrm{~Hz}, 5-14 \mathrm{~Hz}, 5-18 \mathrm{~Hz}$, and all available frequencies) with a fixed number of 50 iterations are used for the multiscale FWI. Figure 11a presents the inverted result of the multiscale FWI, which shows many cycle-skipping artifacts, and the inversion seems to converge to one of the local minima. Figure $11 \mathrm{~b}$ demonstrates the inverted velocity model after 50 iterations using 
the proposed LCFTI. The overall trend of the long wavelength structures is well matched to the true velocity at shallow depths.

We also use the long-offset data from a shot to illustrate the changes of the crosscorrelation and calculated data during the LCFTI iterations as long-offset data are prone to cycle-skipping. The autocorrelation and observed trace act as the reference values. Figure $12 \mathrm{a}$ and $12 \mathrm{~b}$ present the crosscorrelation and calculated trace from the initial model, respectively, Figure $12 \mathrm{c}$ and $12 \mathrm{~d}$ correspond the crosscorrelation and calculated trace at the tenth iteration, respectively, and Figure $12 \mathrm{e}$ and $12 \mathrm{f}$ show the final crosscorrelation and calculated trace, respectively. We can see that as the number of iterations increases, the crosscorrelation approaches to the autocorrelation. At the same time, the excessive traveltime shifts between the observed and initial calculated trace, which cause severe cycle-skipping artifacts in multiscale FWI (Figure 11a), have been reduced significantly.

We then apply multiscale FWI to the LCFTI inverted result (Figure 11b). We use the same procedure to decompose the data into four different scales and fix the number of iterations at 50. Figure $11 \mathrm{c}$ shows that our proposed method can provide a good initial model for conventional FWI to obtain a high-resolution shallow subsurface velocity model. To verify the accuracy of the reconstructed velocity model, a comparison of several traces is given in Figure 13a. The LCFTI approach can succeed with a great traveltime shift, and the final FWI inverted data is well matched with the observed data. Vertical profiles at $\mathrm{z}=0.6 \mathrm{~km}$, shown in Figure 13b, indicate that the proposed method correctly recovers the velocities in the shallow part. 


\section{Field data application}

A 2D shallow marine data set is used to test the performance of the LCFTI approach in a real case. We select 80 shot gathers with $112.5 \mathrm{~m}$ intervals, each of which consists of 300 hydrophones spaced at $12.5 \mathrm{~m}$. The offset range varies from $125 \mathrm{~m}$ to $3862.5 \mathrm{~m}$. Several preprocessing steps are applied to the data, including swell-noise attenuation, surface-related multiple eliminations, and removal of events ahead of the first arrival. The preprocessed shot record displayed in Figure 14a shows that the water depth is very shallow and the energy of the reflections is strong. In this field data application, we use the proposed LCFTI approach to invert all of the preprocessed data.

Instead of estimating a source wavelet from the field data and filtering the data using other tools, we use a Ricker wavelet with a peak frequency of $15 \mathrm{~Hz}$ as a fixed source. Then, the observed data is filtered to a frequency range of $5-40 \mathrm{~Hz}$ by convolution with the Ricker wavelet. Source-wavelet convolution (Yoon et al., 2012) increases the similarity of the waveform between the calculated and field data, which can improve the convergence of the inversion. The filtered shot record is displayed in Figure 14b, which shows that the main events are preserved for the subsequent inversion. Using the arrival time of the primary events and a constant seawater velocity of $1500 \mathrm{~m} / \mathrm{s}$, we calculate the depth of the seabed and build a 1D linearly increasing initial velocity model, as shown in Figure 15a. We utilize the layer-stripping scheme (Wang and Rao, 2009) to gradually reconstruct the velocity 
model by separating the model into three layers. For each layer, we first use LCFTI to reconstruct the low-wavenumber background and then conduct conventional FWI using the LCFTI result as input. When the misfit is sufficiently small, we freeze the upper layers and move to the next layer. The final inverted velocity model and the calculated data are displayed in Figures $15 \mathrm{~b}$ and $14 \mathrm{c}$, respectively. The shallow subsurface velocity model is clearly improved, and the calculated shot record shows high similarity with the filtered record.

To verify the quality of the inverted result, the migrated images and selected common-image gathers (CIGs) at several spatial locations are generated using the initial model and the inverted model, which are shown in Figures 16a and 16b and Figures $17 \mathrm{a}$, and $17 \mathrm{~b}$, respectively. The migration image using the inverted model is clearly improved, especially for prominent structures (minor fault, anticline) as indicated by arrows and boxes in Figure 16. The corresponding CIGs become flatter, brighter, and more continuous than those from the initial model, as indicated by arrows in Figure 17.

\section{DISCUSSION}

Instead of using only the crosscorrelation like CWTI, we include the autocorrelation as a reference and establish a traveltime-weighted-L2-norm objective function. The objective function avoids the resolution-loss problem observed in the conventional correlation-based objective function, and the corresponding model updates based on the Rytov approximation are always in the right direction. In the 
field data application, we apply small selection windows to the data to localize kinematic errors, which can suppress cross-talk between neighboring arrivals (Diaz and Sava, 2015). However, one limitation of our strategy is that the computational cost required for wavefield calculation using the SEM scheme is slightly higher compared to the conventional finite-difference method.

The LCFTI approach demonstrates robust convergence and very good recoverability in the shallow part (depth less than $1.5 \mathrm{~km}$ ) of the velocity model without low-frequency components or a good initial velocity model, as shown in Figures $11 \mathrm{~b}$ and $15 \mathrm{~b}$. On the other hand, the deep region still suffers from lower resolution, even when using multiscale FWI, as shown in Figure 11c. A possible explanation for this behavior is that the LCFTI algorithm focuses more on matching the traveltime of early arrivals. Similar to various wave-equation traveltime inversion methods (Luo and Schuster, 1991; Zhang et al., 2015; Luo et al., 2016), the LCFTI approach mainly depends on the traveltime information to provide a kinematically accurate model at the cost of lower resolution. More work is needed to improve the resolution and reconstruction capability in the deep region.

\section{CONCLUSION}

The successful implementation of FWI strongly depends upon either a good starting model or low-frequency seismic data, which are not always available in practice. We have successfully reconstructed a good shallow subsurface velocity model without requiring a good starting model or low-frequency seismic data by 
using the LCFTI approach we proposed. We have shown that the LCFTI velocity model can be a good starting model for high-resolution FWI. Unlike the conventional CWTI approach, we designed a least-squares correlation-based objective function that incorporates the autocorrelation to enhance convergence and avoid the resolution-loss issue found in CWTI. We proved that the Rytov approximation is more suitable for the wave-equation traveltime inversion than the Born approximation, and the Rytov-approximation-based LCFTI gradient can provide accurate direction for model updates. We simulated a high-accuracy seismic wavefield using the spectral-element method under the challenge of complex topography. Synthetic and real data examples demonstrated the effectiveness of the proposed LCFTI method. The high-resolution shallow subsurface velocity structure obtained based on our approach can be used for static and depth imaging applications.

\title{
ACKNOWLEDGMENTS
}

We want to thank Anatoly Baumstein, Deyan Draganov and three anonymous reviewers for their helpful comments. We thank the open source spectral-element software package SPECFEM2D for our wavefield computing. The research was funded by the National Natural Science Foundation of China (Grant No. 41730425 and 41430321), and The National Major Project of China (Grant 2017ZX05008-007).

\author{
APPENDIX A \\ BORN-APPROXIMATION-BASED GRADIENT AND ADJOINT SOURCE OF
}




\section{CWTI}

The adjoint-state algorithm (Plessix, 2006) is used to derive the gradient $\chi_{\boldsymbol{B}}$ of CWTI. The gradient of the correlation-based objective function with respect to the slowness model $s\left(\boldsymbol{r}^{\prime}\right)$ can be represented as

$$
\begin{aligned}
\chi_{\boldsymbol{B}}= & \frac{\partial M_{C W T I}}{\partial s\left(\boldsymbol{r}^{\prime}\right)}=\sum_{\boldsymbol{r}_{g}, \boldsymbol{r}_{s}} \int W(\tau)^{2} C_{\text {cross }} \frac{\partial C_{\text {cross }}}{\partial s\left(\boldsymbol{r}^{\prime}\right)} d \tau \\
& =\sum_{\boldsymbol{r}_{g}, \boldsymbol{r}_{s}} \iint W(\tau)^{2} C_{\text {cross }} \frac{\partial p_{c}\left(\boldsymbol{r}_{g}, t ; \boldsymbol{r}_{\boldsymbol{s}}\right)}{\partial s\left(\boldsymbol{r}^{\prime}\right)} p_{o}\left(\boldsymbol{r}_{g}, t+\tau ; \boldsymbol{r}_{s}\right) d \tau d t, \quad(\mathrm{~A}-1)
\end{aligned}
$$

where the Fréchet derivative based on the Born approximation is given by (Luo and Schuster, 1991)

$$
\frac{\partial p_{c}\left(\boldsymbol{r}_{g}, t ; \boldsymbol{r}_{\boldsymbol{s}}\right)}{\partial s\left(\boldsymbol{r}^{\prime}\right)}=-2 s_{0}\left(\boldsymbol{r}^{\prime}\right) G\left(\boldsymbol{r}_{g}, t ; \boldsymbol{r}^{\prime}\right) * \ddot{p}_{c}\left(\boldsymbol{r}^{\prime}, t ; \boldsymbol{r}_{s}\right)
$$

with $s_{0}$ denoting the background slowness and $G$ representing the Green's function. Substituting the Fréchet derivative, equation A-2, into gradient equation A-1, the gradient can be rewritten as

$$
\chi_{\boldsymbol{B}}=-2 s_{0}\left(\boldsymbol{r}^{\prime}\right) \sum_{\boldsymbol{r}_{g}, \boldsymbol{r}_{s}} \iint W(\tau)^{2} C_{c r o s s} p_{o}\left(\boldsymbol{r}_{g}, t+\tau ; \boldsymbol{r}_{s}\right) G\left(\boldsymbol{r}_{g}, t ; \boldsymbol{r}^{\prime}\right) * \ddot{p}_{c}\left(\boldsymbol{r}^{\prime}, t ; \boldsymbol{r}_{s}\right) d \tau d t
$$

Utilizing the identity

$$
\int[G(t) * f(t)] h(t) d t=\int f(t)[G(-t) * h(t)] d t
$$

and the temporal reciprocity of the Green's function

$$
G\left(\boldsymbol{r},-t ; \boldsymbol{r}^{\prime}, 0\right)=G\left(\boldsymbol{r}, 0 ; \boldsymbol{r}^{\prime}, t\right)
$$

the gradient can be rewritten as

$$
\chi_{\boldsymbol{B}}=-2 s_{0}\left(\boldsymbol{r}^{\prime}\right) \sum_{\boldsymbol{r}_{g}, \boldsymbol{r}_{s}} \int \ddot{p}_{c}\left(\boldsymbol{r}^{\prime}, t ; \boldsymbol{r}_{s}\right) G\left(\boldsymbol{r}_{g}, 0 ; \boldsymbol{r}^{\prime}, t\right)
$$




$$
\begin{aligned}
& * \int W(\tau)^{2} C_{\text {cross }} p_{o}\left(\boldsymbol{r}_{g}, t+\tau ; \boldsymbol{r}_{s}\right) d \tau d t \\
&=-2 s_{0}\left(\boldsymbol{r}^{\prime}\right) \sum_{\boldsymbol{r}_{g}, \boldsymbol{r}_{s}} \int \ddot{p}_{c}\left(\boldsymbol{r}^{\prime}, t ; \boldsymbol{r}_{s}\right) P_{B}^{\prime} d t,
\end{aligned}
$$

where $P_{B}^{\prime}$ denotes the back-propagated wavefield. The adjoint source $\boldsymbol{\delta}_{\boldsymbol{B}}$ is given as

$$
\boldsymbol{\delta}_{\boldsymbol{B}}=\int W(\tau)^{2} C_{\text {cross }} p_{o}\left(\boldsymbol{r}_{g}, t+\tau ; \boldsymbol{r}_{s}\right) d \tau
$$

The adjoint source can be interpreted as the observed data shifted by the traveltime difference between the calculated and observed data, which is measured by the crosscorrelation.

\section{APPENDIX B}

\section{FRÉCHET DERIVATIVE BASED ON THE RYTOV APPROXIMATION}

The Rytov scattering equation (Stork, 1994) in the frequency domain is formulated as

$$
p_{c}\left(\boldsymbol{r}_{g}, \omega ; \boldsymbol{r}_{s}\right) \Delta \Psi=-2 \int s_{0}\left(\boldsymbol{r}^{\prime}\right) G\left(\boldsymbol{r}_{g}, \omega ; \boldsymbol{r}^{\prime}\right) \ddot{p}_{c}\left(\boldsymbol{r}^{\prime}, \omega ; \boldsymbol{r}_{s}\right) \Delta s\left(\boldsymbol{r}^{\prime}\right) d \boldsymbol{r}^{\prime}, \quad(B-1)
$$

where $\Delta \Psi$ is the complex wavefield-phase perturbation and $\Delta s$ denotes the model perturbation. Substituting the imaginary part of equation 6 into equation B-1 and then transforming back to the time domain, we obtain a linear relation between the model perturbation and the traveltime shift:

$$
\dot{p}_{c}\left(\boldsymbol{r}_{g}, t ; \boldsymbol{r}_{s}\right) \Delta \tau=-2 \int s_{0}\left(\boldsymbol{r}^{\prime}\right) G\left(\boldsymbol{r}_{g}, t ; \boldsymbol{r}^{\prime}\right) * \ddot{p}_{c}\left(\boldsymbol{r}^{\prime}, t ; \boldsymbol{r}_{s}\right) \Delta s\left(\boldsymbol{r}^{\prime}\right) d \boldsymbol{r}^{\prime} . \quad(B-2)
$$

Multiplying both sides of the linear relation in equation B-2 with $\dot{p}_{c}$ and then normalizing with $\dot{p}_{c} \cdot \dot{p}_{c}$, we obtain the Fréchet derivative with respect to $s\left(\boldsymbol{r}^{\prime}\right)$ : 


$$
\frac{\partial \Delta \tau}{\partial s\left(\boldsymbol{r}^{\prime}\right)}=-2 s_{0}\left(\boldsymbol{r}^{\prime}\right) \frac{\int \dot{p}_{c}\left(\boldsymbol{r}_{g}, t ; \boldsymbol{r}_{s}\right) G\left(\boldsymbol{r}^{\prime}, t ; \boldsymbol{r}_{g}\right) * \ddot{p}_{c}\left(\boldsymbol{r}^{\prime}, t ; \boldsymbol{r}_{s}\right) d t}{\int \dot{p}_{c}\left(\boldsymbol{r}_{g}, t ; \boldsymbol{r}_{s}\right) \dot{p}_{c}\left(\boldsymbol{r}_{g}, t ; \boldsymbol{r}_{s}\right) d t} .
$$

Under the assumption that a velocity perturbation causes only traveltime shift, we can use $p_{o}\left(\boldsymbol{r}_{g}, t+\tau ; \boldsymbol{r}_{s}\right)$ to replace $p_{c}\left(\boldsymbol{r}_{g}, t ; \boldsymbol{r}_{s}\right)$ (equation 7). Then, the Fréchet derivative based on the Rytov approximation can be represented as

$$
\frac{\partial \Delta \tau}{\partial s\left(\boldsymbol{r}^{\prime}\right)}=-2 s_{0}\left(\boldsymbol{r}^{\prime}\right) \frac{\int \dot{p}_{o}\left(\boldsymbol{r}_{g}, t+\tau ; \boldsymbol{r}_{s}\right) G\left(\boldsymbol{r}^{\prime}, t ; \boldsymbol{r}_{g}\right) * \ddot{p}_{c}\left(\boldsymbol{r}^{\prime}, t ; \boldsymbol{r}_{s}\right) d t}{\int \dot{p}_{o}\left(\boldsymbol{r}_{g}, t+\tau ; \boldsymbol{r}_{s}\right) \dot{p}_{o}\left(\boldsymbol{r}_{g}, t+\tau ; \boldsymbol{r}_{s}\right) d t} \quad(\mathrm{~B}-4)
$$

\section{APPENDIX C}

\section{GRADIENT AND ADJOINT SOURCE OF LCFTI}

Recall that the gradient of the proposed LCFTI approach with respect to the slowness $s\left(\boldsymbol{r}^{\prime}\right)$, which is based on the Rytov approximation, is given as

$$
\chi_{\boldsymbol{R}}=\frac{\partial M_{L C F T I}}{\partial s\left(\boldsymbol{r}^{\prime}\right)}=\sum_{\boldsymbol{r}_{g}, \boldsymbol{r}_{s}} \int W(\tau)^{2}\left(C_{\text {auto }}-C_{\text {cross }}\right)\left[-\frac{\partial C_{\text {cross }}}{\partial \tau} \frac{\partial \Delta \tau}{\partial s\left(\boldsymbol{r}^{\prime}\right)}\right] d \tau . \quad(\mathrm{C}-1)
$$

Using integration by parts, the gradient becomes

$$
\begin{aligned}
\chi_{\boldsymbol{R}}=\sum_{\boldsymbol{r}_{g}, \boldsymbol{r}_{s}}[ & -W(\tau)^{2}\left(C_{\text {auto }}-C_{\text {cross }}\right) C_{\text {cross }} \tau_{\tau=-\tau_{\max }}^{\tau=\tau_{\max }} \\
& \left.+\int 2 W(\tau)\left(C_{\text {auto }}-C_{\text {cross }}\right) C_{\text {cross }}\right] \frac{\partial \Delta \tau}{\partial s\left(\boldsymbol{r}^{\prime}\right)} d \tau .
\end{aligned}
$$

Usually, $\tau_{\max }$ is the maximum possible traveltime shift; thus, the correlation will go to zero when $\tau=\tau_{\max }$ and $\tau=-\tau_{\max }$. Therefore, the gradient in equation $\mathrm{C}-2$ can be represented as

$$
\chi_{R}=\sum_{r_{g}, r_{s}} \int 2 W(\tau)\left(C_{\text {auto }}-C_{\text {cross }}\right) C_{\text {cross }} \frac{\partial \Delta \tau}{\partial s\left(\boldsymbol{r}^{\prime}\right)} d \tau
$$

Substituting the Fréchet derivative based on the Rytov approximation, equation B-4, 
into the gradient in equation C-3, we obtain the LCFTI gradient as

$$
\begin{aligned}
\chi_{\boldsymbol{R}}=-4 s_{0}\left(\boldsymbol{r}^{\prime}\right) \sum_{\boldsymbol{r}_{g}, \boldsymbol{r}_{s}} \int W(\tau)\left(C_{\text {auto }}-C_{\text {cross }}\right) C_{\text {cross }} \\
\frac{\int \dot{p}_{o}\left(\boldsymbol{r}_{g}, t+\tau ; \boldsymbol{r}_{s}\right) G\left(\boldsymbol{r}^{\prime}, t ; \boldsymbol{r}_{g}\right) * \ddot{p}_{c}\left(\boldsymbol{r}^{\prime}, t ; \boldsymbol{r}_{s}\right) d t}{\int \dot{p}_{o}\left(\boldsymbol{r}_{g}, t+\tau ; \boldsymbol{r}_{s}\right) \dot{p}_{o}\left(\boldsymbol{r}_{g}, t+\tau ; \boldsymbol{r}_{s}\right) d t} d \tau .
\end{aligned}
$$

Finally, utilizing the identities of equations A-4 and A-5, the LCFTI gradient can be rewritten as

$$
\chi_{\boldsymbol{R}}=-4 s_{0}\left(\boldsymbol{r}^{\prime}\right) \sum_{\boldsymbol{r}_{g}, \boldsymbol{r}_{s}} \int \ddot{p}_{c}\left(\boldsymbol{r}^{\prime}, t ; \boldsymbol{r}_{s}\right) \cdot P_{R}^{\prime} d t
$$

where $P_{R}^{\prime}$ is the back-propagated wavefield, and the adjoint source is given by

$$
\boldsymbol{\delta} \boldsymbol{s}_{\boldsymbol{R}}=\int W(\tau) \frac{\left(C_{\text {auto }}-C_{\text {cross }}\right) \cdot C_{\text {cross }} \cdot \dot{p}_{o}\left(\boldsymbol{r}_{g}, t+\tau ; \boldsymbol{r}_{s}\right)}{\int \dot{p}_{o}\left(\boldsymbol{r}_{g}, t+\tau ; \boldsymbol{r}_{s}\right) \dot{p}_{o}\left(\boldsymbol{r}_{g}, t+\tau ; \boldsymbol{r}_{s}\right) d t} d \tau
$$

Compared with the adjoint source of the conventional CWTI (equation A-7), the adjoint source of the LCFTI can be interpreted as the observed wavefield shifted by the traveltime difference, which is measured by both autocorrelation and crosscorrelation. This key point makes the gradient and misfit go to zero when the inverted model matches the correct model.

\section{REFERENCES}

Adamczyk, A., M. Malinowski, and A. Malehmir, 2013, Application of first-arrival tomography to characterize a quick clay landslide site in Southwest Sweden: Acta Geophysica, 61, no. 5, 1057-1073, doi: 10.2478/s11600-013-0136-y.

Adamczyk, A., M. Malinowski, and A. Malehmir, 2014, High-resolution near-surface velocity model building using full-waveform inversion - a case study from 
southwest Sweden: Geophysical Journal International, 197, no. 3, 1693-1704, doi: $10.1093 / \mathrm{gji} / \mathrm{ggu} 070$.

Almomin, A., and B. Biondi, 2012, Tomographic full waveform inversion: Practical and computationally feasible approach: 82nd Annual International Meeting, SEG, Expanded Abstracts, 1-5, doi: 10.1190/segam2012-0976.1.

Bergamo, P., D. Boiero, and L. V. Socco, 2012, Retrieving 2D structures from surface-wave data by means of space-varying spatial windowing: Geophysics, 77, no. 4, EN39-EN51, doi: 10.1190/geo2012-0031.1.

Boiero, D., and L. V. Socco, 2010, Retrieving lateral variations from surface wave dispersion curves: Geophysical Prospecting, 58, no. 6, 977-996, doi: 10.1111/j.1365-2478.2010.00877.x.

Bunks, C., F. M. Saleck, S. Zaleski, and G. Chavent, 1995, Multiscale seismic waveform inversion: Geophysics, 60, no. 5, 1457-1473, doi: 10.1190/1.1443880.

Chi, B., Y. Wang, Y. Liu, and L. Dong, 2012, Hybrid Born and Rytov scattering series and its application in full waveform inversion: 82nd Annual International Meeting, 1-5, doi: 10.1190/segam2012-0934.1.

Choi, Y., and T. Alkhalifah, 2015, Unwrapped phase inversion with an exponential damping: Geophysics, 80, no. 5, R251-R264, doi: 10.1190/geo2014-0498.1.

Choi, Y., and T. Alkhalifah, 2016, An optimized correlation-based full waveform inversion: 78th Annual International Conference and Exhibition, EAGE, Extended Abstracts, Tu P1 13, doi: 10.3997/2214-4609.201600642. 
Clayton, R. W., and R. H. Stolt, 1981, A Born - WKBJ inversion method for acoustic reflection data: Geophysics, 46, no. 11, 1559-1567, doi: 10.1190/1.1441162.

de Hoop, M. V., and R. D. van der Hilst, 2005, On sensitivity kernels for 'wave-equation' transmission tomography: Geophysical Journal International, 160, no. 2, 621-633, doi: 10.1111/j.1365-246X.2004.02509.x.

Diaz, E., and P. Sava, 2015, Data domain wavefield tomography using local correlation functions: 85th Annual International Meeting, SEG, Expanded Abstracts, 1361-1365, doi: 10.1190/segam2015-5881732.1.

Fichtner, A. 2011, Full seismic waveform modelling and inversion: Springer Science \& Business Media, doi: 10.1007/978-3-642-15807-0.

Gray, S. H., and K. J. Marfurt, 1995, Migration from topography: Improving the near-surface image: Canadian journal of exploration geophysics, 31, 18-24.

Komatitsch, D., and J. P. Vilotte, 1998, The spectral element method: An efficient tool to simulate the seismic response of $2 \mathrm{~d}$ and $3 \mathrm{~d}$ geological structures: Bulletin of the Seismological Society of America, 88, 368-392.

Lanz, E., H. Maurer, and A. G. Green, 1998, Refraction tomography over a buried waste disposal site: Geophysics, 63, no. 4, 1414-1433, doi: 10.1190/1.1444443.

Liu, Y., B. He, H. Lu, Z. Zhang, X.-B. Xie, and Y. Zheng, 2018, Full-intensity waveform inversion: Geophysics, 83, no. 6, R649-R658, doi: 10.1190/geo2017-0682.1.

Liu, Y., J. Teng, H. Lan, X. Si, and X. Ma, 2014, A comparative study of finite element and spectral element methods in seismic wavefield modeling: 
Geophysics, 79, no. 2, T91-T104, doi: 10.1190/geo2013-0018.1.

Liu, Z., and Y. Zheng, 2015, Direct waveform inversion: 85th Annual International Meeting, SEG, Expanded Abstract, 1268-1273, doi: 10.1190/segam2015-5923910.1.

Luo, S., and P. Sava, 2011, A deconvolution-based objective function for wave-equation inversion: 81st Annual International Meeting, SEG, Expanded Abstracts, 2788-2792, doi: 10.1190/1.3627773.

Luo, Y., Y. Ma, Y. Wu, H. Liu, and L. Cao, 2016, Full-traveltime inversion: Geophysics, 81, no. 5, R261-R274, doi: 10.1190/geo2015-0353.1.

Luo, Y., and G. T. Schuster, 1991, Wave - equation traveltime inversion: Geophysics, 56, no. 5, 645-653, doi: 10.1190/1.1443081.

Ma, Y., and D. Hale, 2013, Wave-equation reflection traveltime inversion with dynamic warping and full-waveform inversion: Geophysics, 78, no. 6, R223-R233, doi: 10.1190/geo2013-0004.1.

Martí, D., R. Carbonell, I. Flecha, I. Palomeras, J. Font-Capó, E. Vázquez-Suñé, and A. Pérez-Estaún, 2008, High-resolution seismic characterization in an urban area: Subway tunnel construction in Barcelona, Spain: Geophysics, 73, no. 2, B41-B50, doi: 10.1190/1.2832626.

Masoni, I., J.-L. Boelle, R. Brossier, and J. Virieux, 2016, Layer stripping FWI for surface waves: 86th Annual International Meeting, SEG, Expanded Abstract, 1369-1373, doi: 10.1190/segam2016-13859781.1.

Mi, B. B., J. H. Xia, C. Shen, L. M. Wang, Y. Hu, and F. Cheng, 2017, Horizontal 
resolution of multichannel analysis of surface waves: Geophysics, 82, no. 3, En51-En66, doi: 10.1190/Geo2016-0202.1.

Park, C. B., R. D. Miller, and J. Xia, 1999, Multichannel analysis of surface waves: Geophysics, 64, no. 3, 800-808, doi: 10.1190/1.1444590.

Pérez Solano, C. A., D. Donno, and H. Chauris, 2014, Alternative waveform inversion for surface wave analysis in 2-D media: Geophysical Journal International, 198, no. 3, 1359-1372, doi: 10.1093/gji/ggu211.

Pérez Solano, C. A., D. Donno, and H. Chauris, 2016, Finite-difference strategy for elastic wave modelling on curved staggered grids: Computational Geosciences, 20, no. 1, 245-264, doi: 10.1007/s10596-016-9561-8.

Plessix, R. E., 2006, A review of the adjoint-state method for computing the gradient of a functional with geophysical applications: Geophysical Journal International, 167, no. 2, 495-503, doi: 10.1111/j.1365-246X.2006.02978.x.

Rajan, S. D., and G. V. Frisk, 1989, A comparison between the Born and Rytov approximations for the inverse backscattering problem: Geophysics, 54, no. 7, 864-871, doi: 10.1190/1.1442715.

Sacchi, M. D., and T. J. Ulrych, 1995, High - resolution velocity gathers and offset space reconstruction: Geophysics, 60, no. 4, 1169-1177, doi: $10.1190 / 1.1443845$.

Shen, P., and W. W. Symes, 2008, Automatic velocity analysis via shot profile migration: Geophysics, 73, no. 5, VE49-VE59, doi: 10.1190/1.2972021.

Shen, X., 2010, Near-surface velocity estimation by weighted early-arrival waveform 
inversion: 80th Annual International Meeting, SEG, Expanded Abstracts, 1975-1979, doi: 10.1190/1.3513230.

Sheng, J., A. Leeds, M. Buddensiek, and G. T. Schuster, 2006, Early arrival waveform tomography on near-surface refraction data: Geophysics, 71, no. 4, U47-U57, doi: 10.1190/1.2210969.

Shin, C., and Y. H. Cha, 2008, Waveform inversion in the Laplace domain: Geophysical Journal International, 173, no. 3, 922-931, doi: 10.1111/j.1365-246X.2008.03768.x.

Shin, C., and Y. H. Cha, 2009, Waveform inversion in the Laplace-Fourier domain: Geophysical Journal International, 177, no. 3, 1067-1079, doi: 10.1111/j.1365-246X.2009.04102.x.

Stork, C., 1994, Hybrid Born and Rytov inversion: 64th Annual International Meeting, SEG, Expanded Abstracts, 1008-1012, doi: 10.1190/1.1822682.

Tarrass, I., L. Giraud, and P. Thore, 2011, New curvilinear scheme for elastic wave propagation in presence of curved topography: Geophysical Prospecting, 59, no. 5, 889-906, doi: 10.1111/j.1365-2478.2011.00972.x.

van Leeuwen, T., and W. A. Mulder, 2010, A correlation-based misfit criterion for wave-equation traveltime tomography: Geophysical Journal International, 182, no. 3, 1383-1394, doi: 10.1111/j.1365-246X.2010.04681.x.

Virieux, J., and S. Operto, 2009, An overview of full-waveform inversion in exploration geophysics: Geophysics, 74, no. 6, WCC1-WCC26, doi: 10.1190/1.3238367. 
Wang, Y., and Y. Rao, 2009, Reflection seismic waveform tomography: Journal of Geophysical Research, 114, no. B3, doi: 10.1029/2008jb005916.

Warner, M., and L. Guasch, 2016, Adaptive waveform inversion: Theory: Geophysics, 81, no. 6, R429-R445, doi: 10.1190/geo2015-0387.1.

Wu, R.-S., J. Luo, and B. Wu, 2014, Seismic envelope inversion and modulation signal model: Geophysics, 79, no. 3, WA13-WA24, doi: 10.1190/geo2013-0294.1.

Wu, Z., and T. Alkhalifah, 2018, Selective data extension for full-waveform inversion: An efficient solution for cycle skipping: Geophysics, 83, no. 3, R201-R211, doi: 10.1190/geo2016-0649.1.

Yi, J., and Y. Liu, 2017, A new least-square correlation-based near surface full traveltime inversion: 87th Annual International Meeting, SEG, Expanded Abstract, 1314-1318, doi: 10.1190/segam2017-17673580.1.

Yoon, K., S. Suh, J. Cai, and B. Wang, 2012, Improvements in time domain FWI and its applications: 82nd Annual International Meeting, SEG, Expanded Abstracts, 1-5, doi: 10.1190/segam2012-1535.1.

Yuan, Y. O., F. J. Simons, and E. Bozdag, 2015, Multiscale adjoint waveform tomography for surface and body waves: Geophysics, 80, no. 5, R281-R302, doi: 10.1190/Geo2014-0461.1.

Zhang, S., Y. Luo, and G. Schuster, 2015, Shot- and angle-domain wave-equation traveltime inversion of reflection data: Synthetic and field data examples: Geophysics, 80, no. 4, S79-S92, doi: 10.1190/geo2014-0223.1. 
Zhang, W., and X. F. Chen, 2006, Traction image method for irregular free surface boundaries in finite difference seismic wave simulation: Geophysical Journal International, 167, no. 1, 337-353, doi: 10.1111/j.1365-246X.2006.03113.x.

Zhang, Y., and D. Wang, 2009, Traveltime information-based wave-equation inversion: Geophysics, 74, no. 6, WCC27-WCC36, doi: 10.1190/1.3243073.

Zhang, Z., T. Alkhalifah, and Z. Wu, 2018, Normalized nonzero-lag crosscorrelation elastic full waveform inversion: 80th Annual International Conference and Exhibition, EAGE, Extended Abstracts, Tu P3 12, doi: $10.3997 / 2214-4609.201800889$

Zhang, Z., G. Schuster, Y. Liu, S. M. Hanafy, and J. Li, 2016, Wave equation dispersion inversion using a difference approximation to the dispersion-curve misfit gradient: Journal of Applied Geophysics, 133, 9-15, doi: 10.1016/j.jappgeo.2016.07.019.

Zhou, H., L. Amundsen, and G. Zhang, 2012, Fundamental issues in full waveform inversion: 81st Annual International Meeting, SEG, Expanded Abstracts, 1-5, doi: 10.1190/1.3627756.

\section{CAPTION LIST}

Figure 1. (a) Observed and calculated traces, and (b) crosscorrelation between the observed and calculated traces, and autocorrelation of the observed trace.

Figure 2. (a) Gaussian-circle inclusion velocity model, and (b) CWTI gradient calculated by using the true velocity model (a) as a trial model. 
Figure 3. (a) Common-shot gather calculated using the true velocity model shown in Figure $2 \mathrm{a}$, and (b) the adjoint source corresponding to this shot gather.

Figure 4. (a) Data misfit with respect to the velocity errors for the proposed LCFTI (black solid) and traditional FWI (gray dashed) using a homogeneous model. (b) Data misfit with respect to the velocity errors for the proposed LCFTI (black solid) and CWTI (black dashed) using the Gaussian-circle inclusion model in Figure 2a.

Figure 5. Comparison of the sensitivity kernels of the proposed LCFTI gradient and Born-approximation-based CWTI gradient by using a lower $(3000 \mathrm{~m} / \mathrm{s})$ and a higher $(5000 \mathrm{~m} / \mathrm{s})$ initial velocity. (a) LCFTI gradient with the lower initial velocity, (b) LCFTI gradient with the higher initial velocity. (c) CWTI gradient with the lower initial velocity, and (d) CWTI gradient with the higher initial velocity. The true homogeneous velocity is $4000 \mathrm{~m} / \mathrm{s}$.

Figure 6. Comparison of the LCFTI and CWTI gradient for the Gaussian-circle inclusion model in Figure 2 a by using a small $(3000 \mathrm{~m} / \mathrm{s})$ and a large $(5000 \mathrm{~m} / \mathrm{s})$ initial Gaussian perturbation. (a) LCFTI gradient with the small initial Gaussian perturbation, (b) LCFTI gradient with the large intial Gaussian perturbation, (c) CWTI gradient with the small initial Gaussian perturbation, and (d) CWTI gradient with the large initial Gaussian perturbation.

Figure 7. (a) The Crosswell velocity model, (b) homogeneous initial velocity model, (c) the inverted velocity using the CWTI, (d) the velocity model inverted using the LCFTI, and (e) the inversion result of traditional FWI using the LCFTI model as a start model. 
Figure 8. Normalized misfit with respect to the iterations for LCFTI (black solid) and CWTI (gray dashed).

Figure 9. (a) The Canadian Foothill velocity model, (b) the homogeneous initial velocity model, and (c) the irregular quadrilateral mesh for SEM, where the grid intervals are enlarged for demonstration.

Figure 10. The deficient data condition: (a) spectrum of the observed data and (b) comparison of the traces between the observed data (red) and calculated data (blue) from the initial model.

Figure 11. Inversion results: (a) a simple multiscale FWI, (b) the proposed LCFTI, and (c) multiscale FWI using the LCFTI result as initial model.

Figure 12. Comparisons of autocorrelation (red) and crosscorrelation (blue), observed (red) and calculated (blue) data for the LCFTI during the iterations: (a) crosscorrelation using the starting model, (b) trace of the starting model, (c) crosscorrelation using the inverted model at the tenth iteration, (d) trace of the inverted model at the tenth iteration, (e) crosscorrelation using the final inverted model, and (f) trace of the final LCFTI model.

Figure 13. (a) Comparison of traces extracted from the observed, initial, LCFTI, and the final FWI seismic data. (b) Comparison of the true model, initial model, LCFTI result and the final inversion result by combing LCFTI with FWI at depth of $0.6 \mathrm{~km}$.

Figure 14. Shot records of (a) the original marine data, (b) the filtered data convolved with the source wavelet, and (c) the synthetic data generated by the final inverted velocity model. 
Figure 15. (a) Initial velocity model, and (b) final inverted velocity model by applying LCFTI and traditional FWI.

Figure 16. Migration images using (a) the initial velocity and (b) the final inverted velocity model. Yellow arrows and boxes indicate the improvements.

Figure17. Selected CIGs at select spatial locations generated using (a) the initial velocity and (b) the final inverted velocity model. Yellow arrows indicate the improvements. 

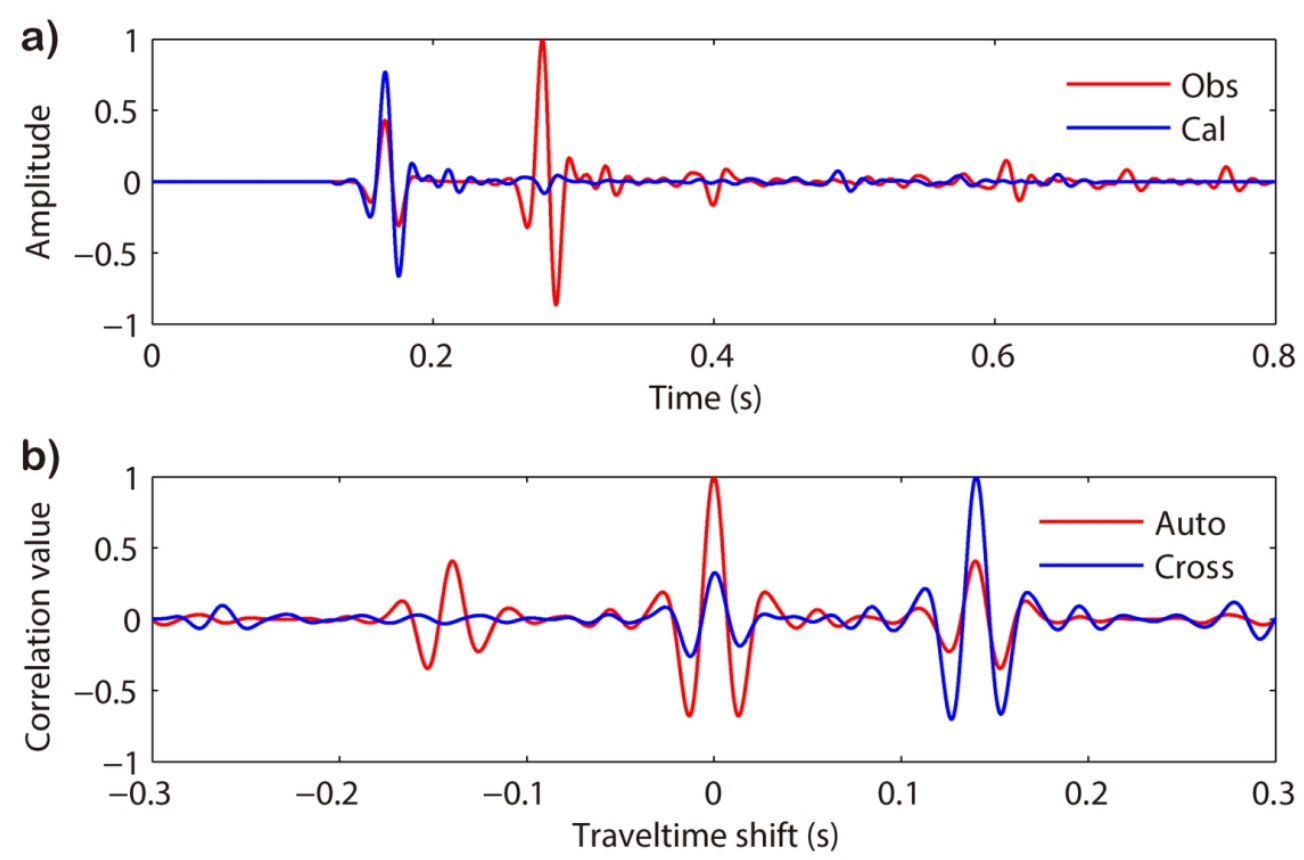

Figure 1. (a) Observed and calculated traces, and (b) crosscorrelation between the observed and calculated traces, and autocorrelation of the observed trace.

$130 \times 88 \mathrm{~mm}(300 \times 300 \mathrm{DPI})$ 

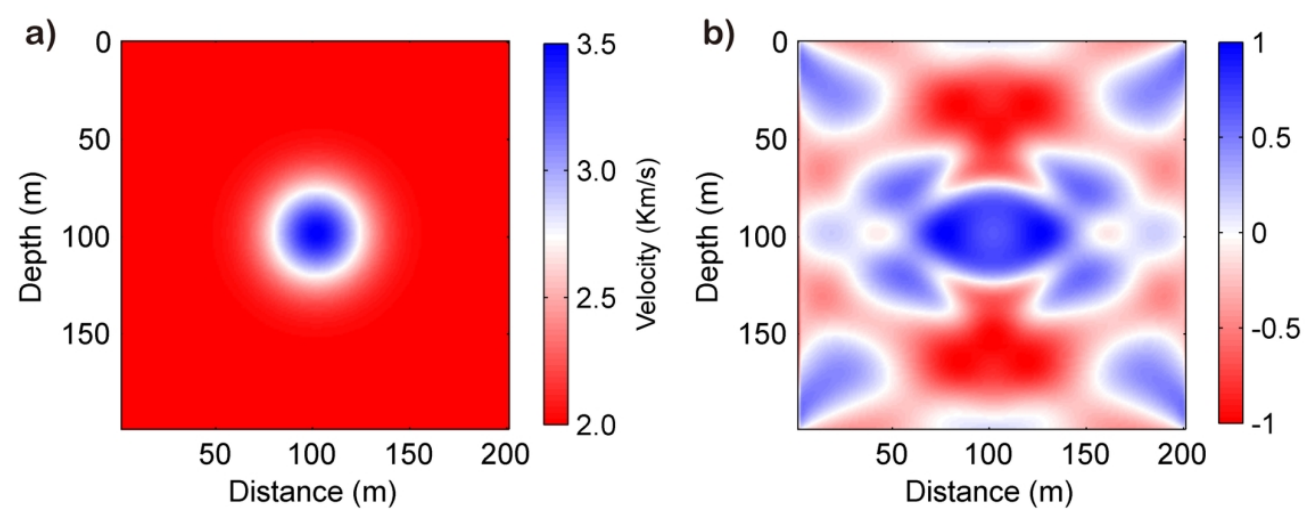

Figure 2. (a) Gaussian-circle inclusion velocity model, and (b) CWTI gradient calculated by using the true velocity model (a) as a trial model.

$$
160 \times 61 \mathrm{~mm}(300 \times 300 \mathrm{DPI})
$$



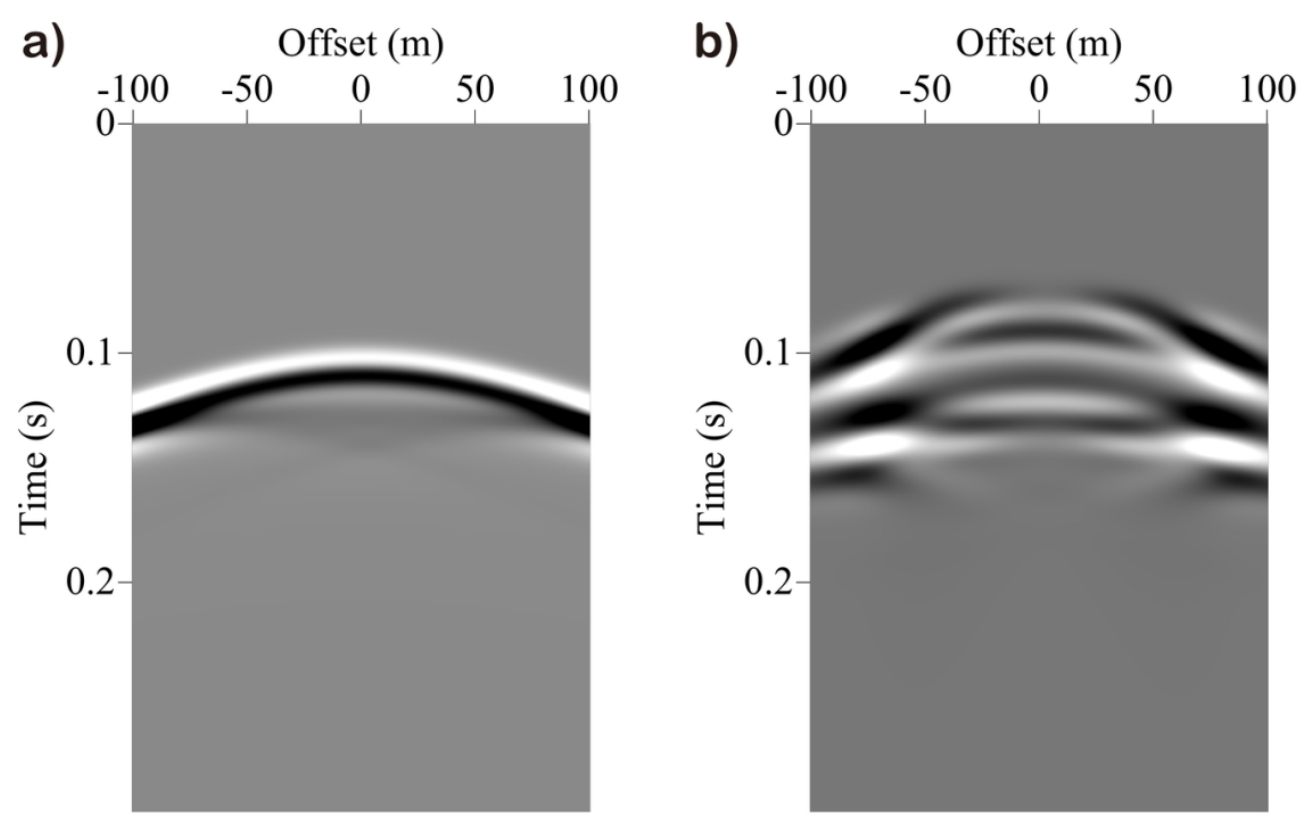

Figure 3. (a) Common-shot gather calculated using the true velocity model shown in Figure $2 a$, and (b) the adjoint source corresponding to this shot gather.

$115 \times 75 \mathrm{~mm}(300 \times 300 \mathrm{DPI})$ 

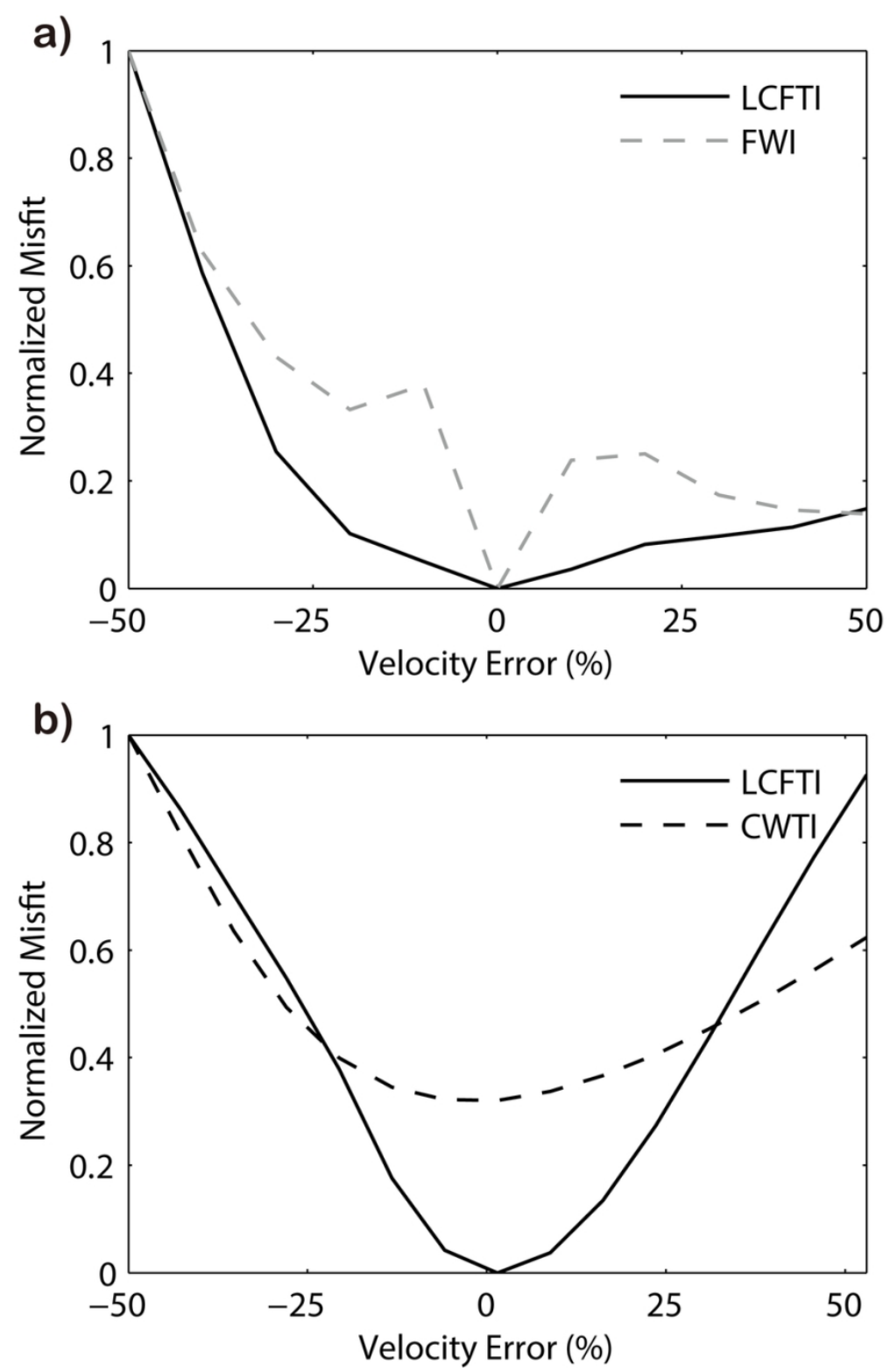

Figure 4. (a) Data misfit with respect to the velocity errors for the proposed LCFTI (black solid) and traditional FWI (gray dashed) using a homogeneous model. (b) Data misfit with respect to the velocity errors for the proposed LCFTI (black solid) and CWTI (black dashed) using the Gaussian-circle inclusion model in Figure 2a.

$89 \times 135 \mathrm{~mm}(300 \times 300 \mathrm{DPI})$ 

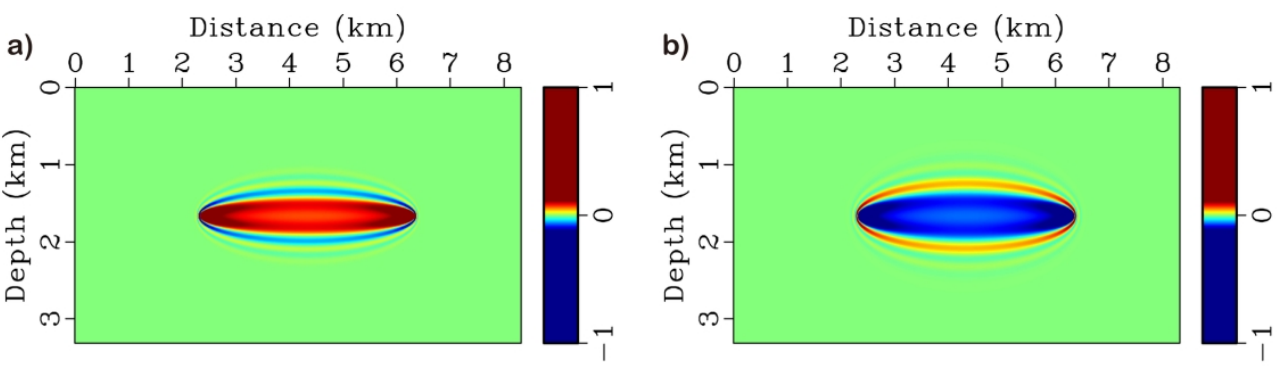

Distance $(\mathrm{km})$
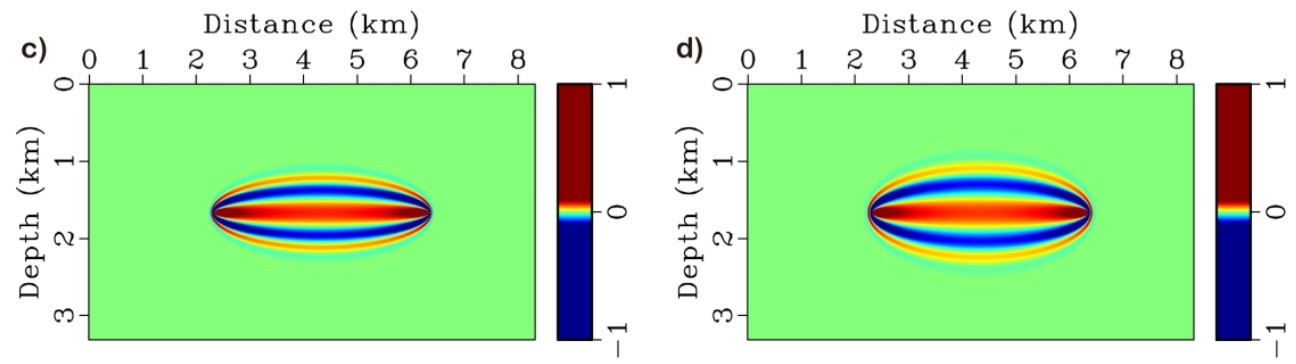

Figure 5. Comparison of the sensitivity kernels of the proposed LCFTI gradient and Born-approximationbased CWTI gradient by using a lower $(3000 \mathrm{~m} / \mathrm{s})$ and a higher $(5000 \mathrm{~m} / \mathrm{s})$ initial velocity. (a) LCFTI gradient with the lower initial velocity, (b) LCFTI gradient with the higher initial velocity. (c) CWTI gradient with the lower initial velocity, and (d) CWTI gradient with the higher initial velocity. The true homogeneous velocity is $4000 \mathrm{~m} / \mathrm{s}$.

$194 \times 111 \mathrm{~mm}(300 \times 300 \mathrm{DPI})$ 
a)

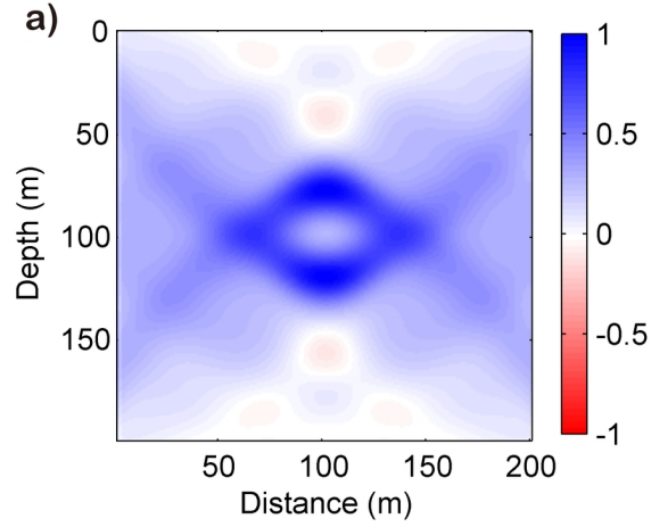

c)

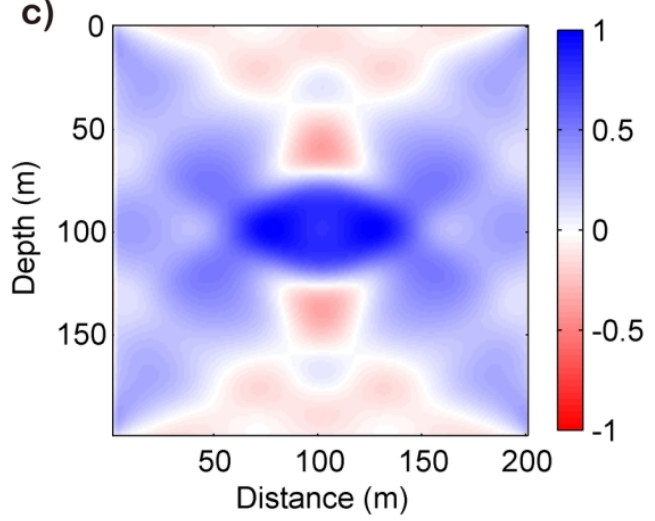

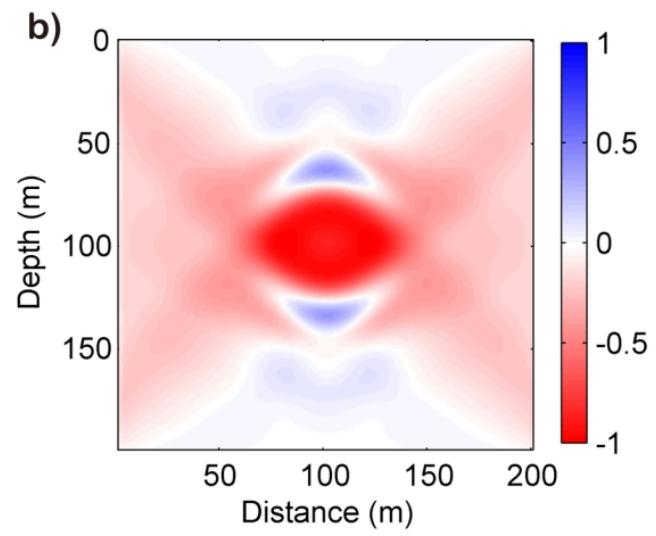

d)

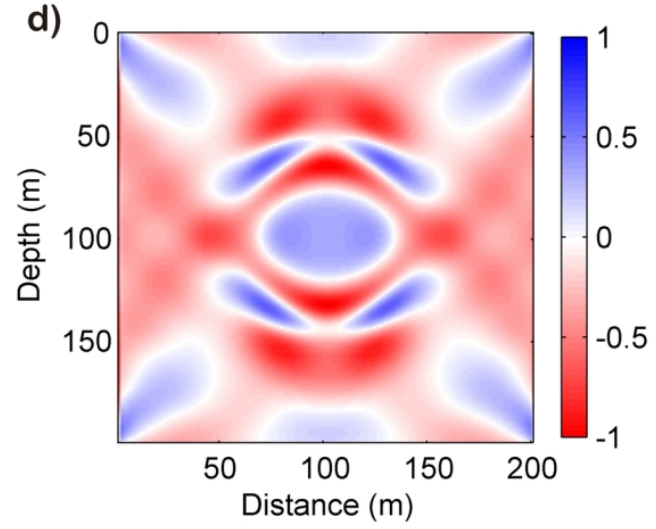

Figure 6. Comparison of the LCFTI and CWTI gradient for the Gaussian-circle inclusion model in Figure 2a by using a small $(3000 \mathrm{~m} / \mathrm{s})$ and a large $(5000 \mathrm{~m} / \mathrm{s})$ initial Gaussian perturbation. (a) LCFTI gradient with the small initial Gaussian perturbation, (b) LCFTI gradient with the large intial Gaussian perturbation, (c) CWTI gradient with the small initial Gaussian perturbation, and (d) CWTI gradient with the large initial Gaussian perturbation.

$152 \times 124 \mathrm{~mm}(300 \times 300$ DPI $)$ 

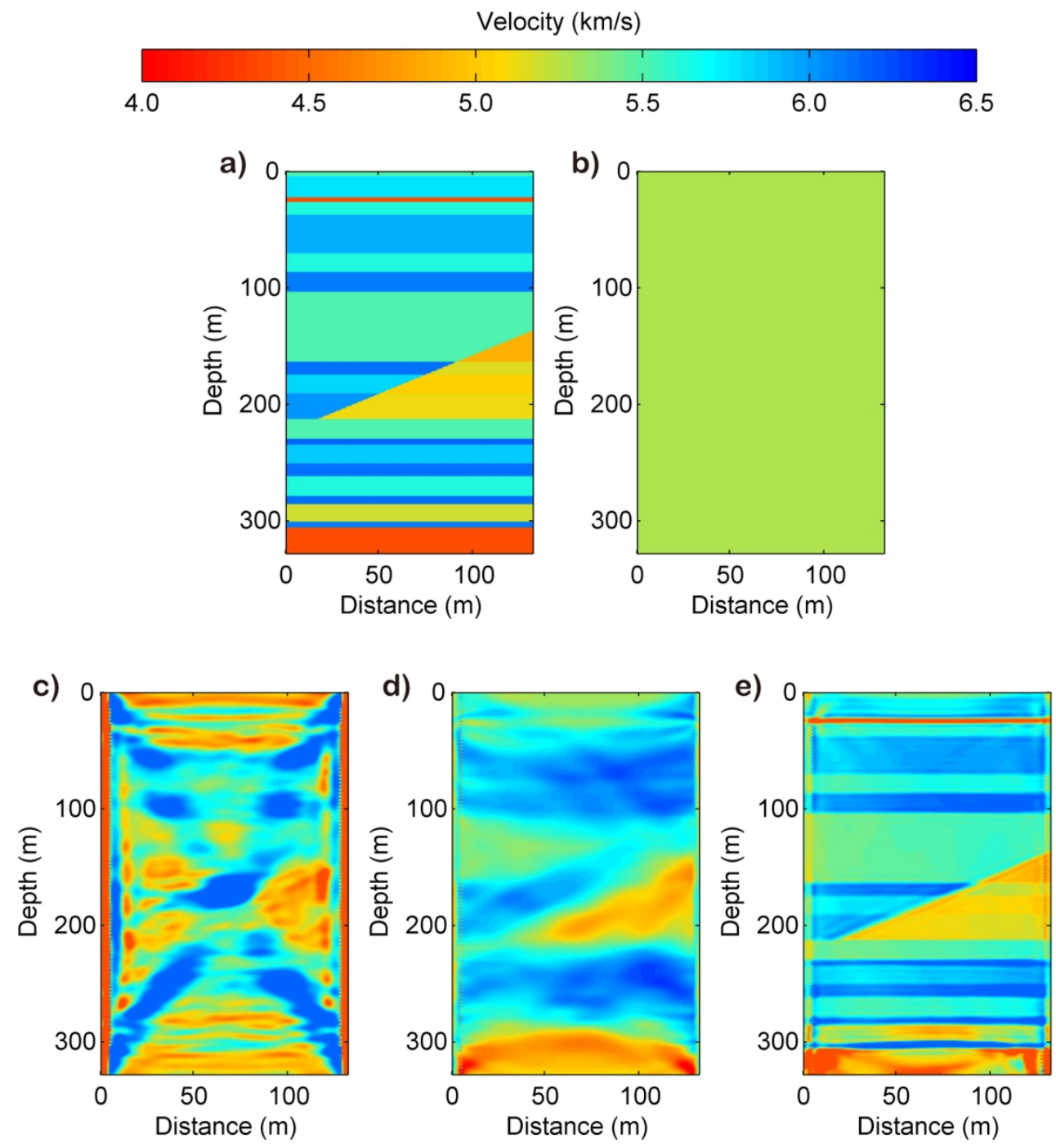

Figure 7. (a) The Crosswell velocity model, (b) homogeneous initial velocity model, (c) the inverted velocity using the CWTI, (d) the velocity model inverted using the LCFTI, and (e) the inversion result of traditional FWI using the LCFTI model as a start model.

$$
151 \times 161 \mathrm{~mm} \text { ( } 300 \times 300 \text { DPI) }
$$




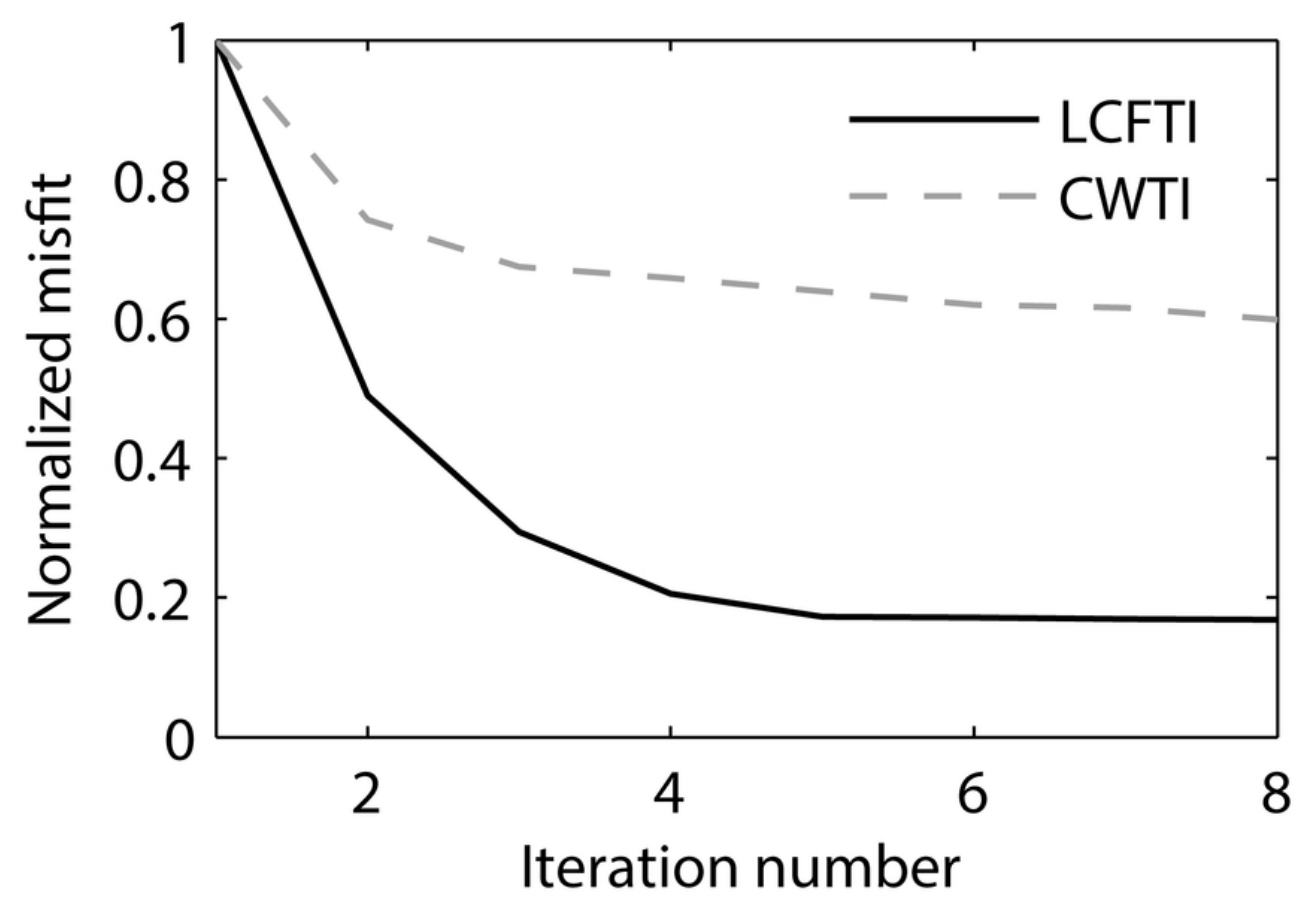

Figure 8. Normalized misfit with respect to the iterations for LCFTI (black solid) and CWTI (gray dashed). $73 \times 53 \mathrm{~mm}(300 \times 300 \mathrm{DPI})$ 

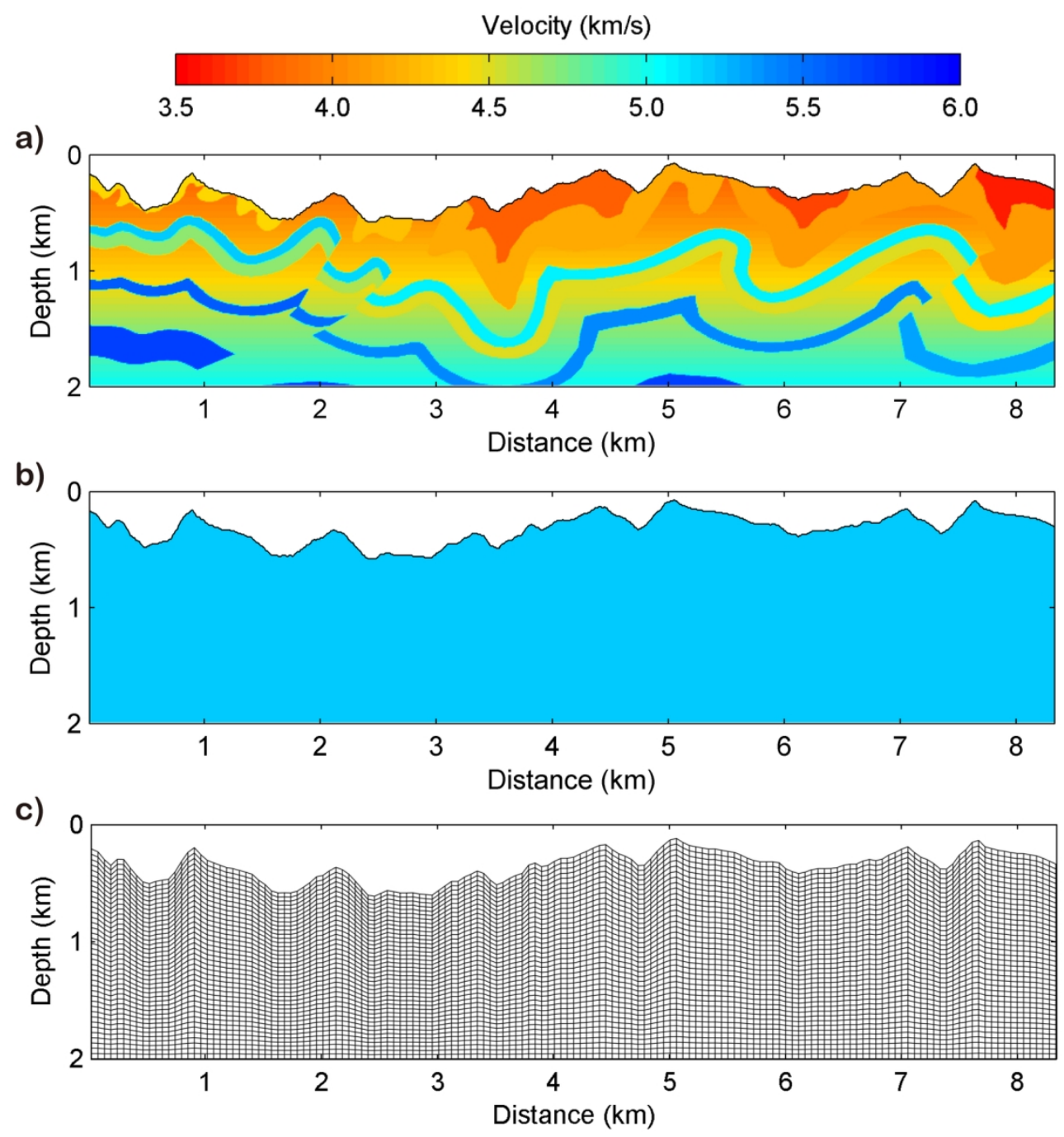

Figure 9. (a) The Canadian Foothill velocity model, (b) the homogeneous initial velocity model, and (c) the irregular quadrilateral mesh for SEM, where the grid intervals are enlarged for demonstration.

$144 \times 150 \mathrm{~mm}(300 \times 300 \mathrm{DPI})$ 
a)

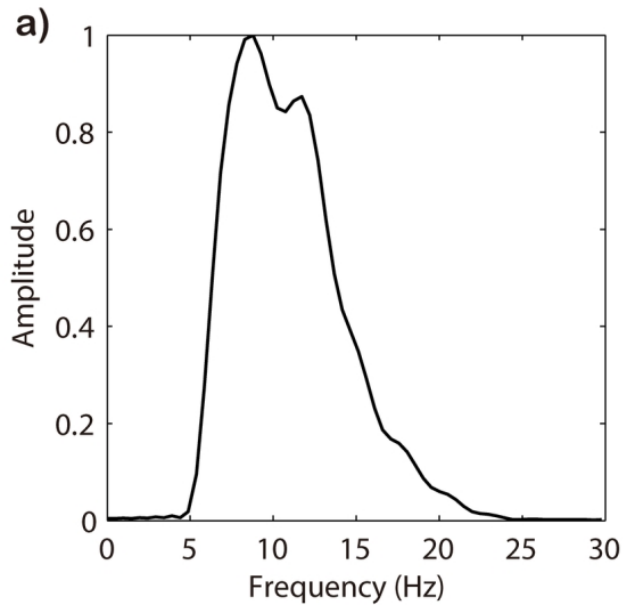

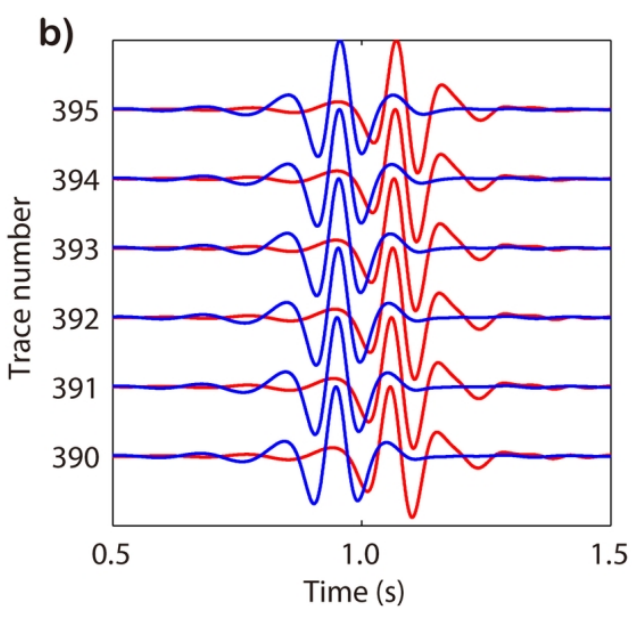

Figure 10. The deficient data condition: (a) spectrum of the observed data and (b) comparison of the traces between the observed data (red) and calculated data (blue) from the initial model.

$146 \times 68 \mathrm{~mm}(300 \times 300 \mathrm{DPI})$ 

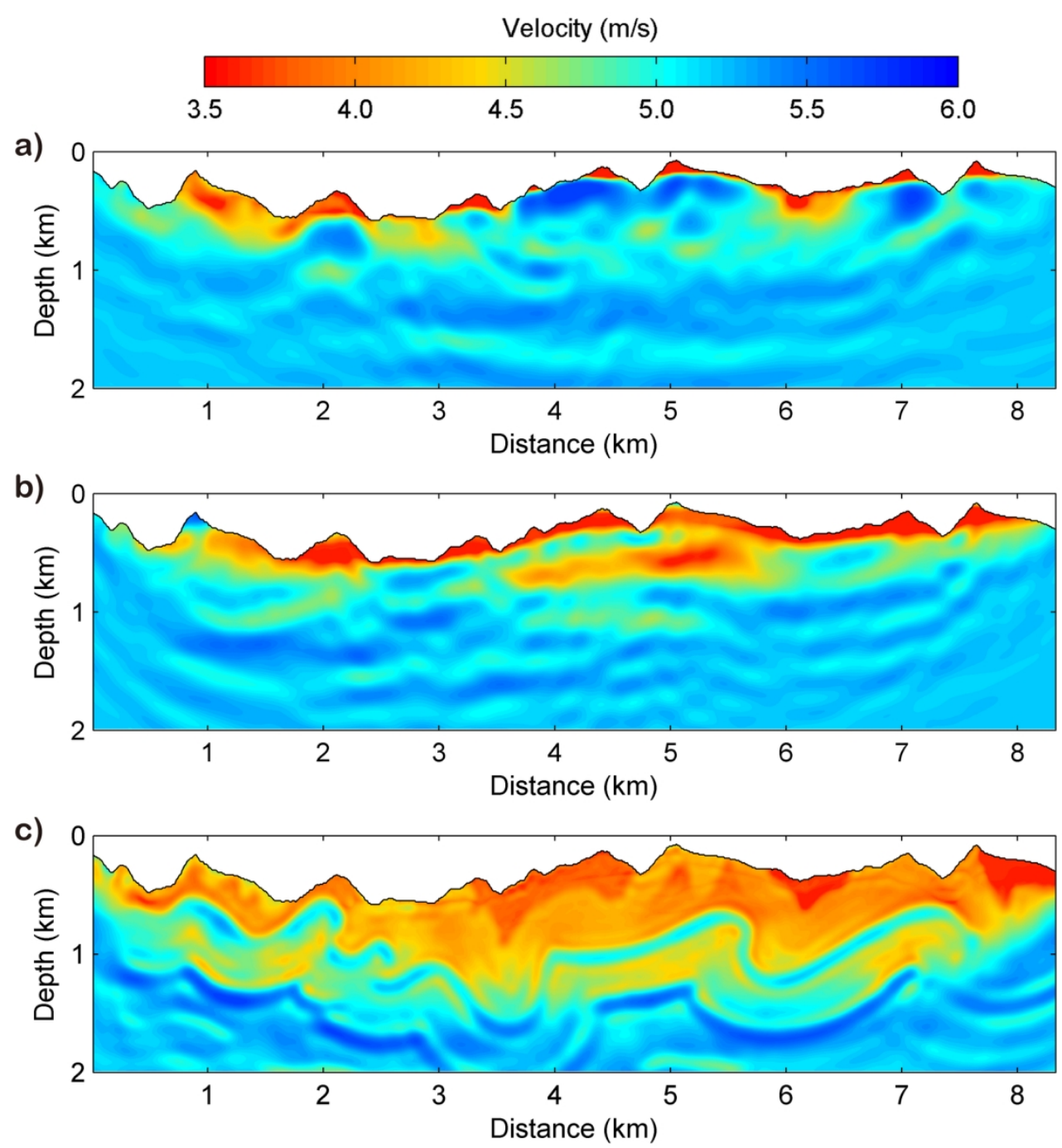

Figure 11. Inversion results: (a) a simple multiscale FWI, (b) the proposed LCFTI, and (c) multiscale FWI using the LCFTI result as initial model.

$145 \times 155 \mathrm{~mm}(300 \times 300 \mathrm{DPI})$ 
a)
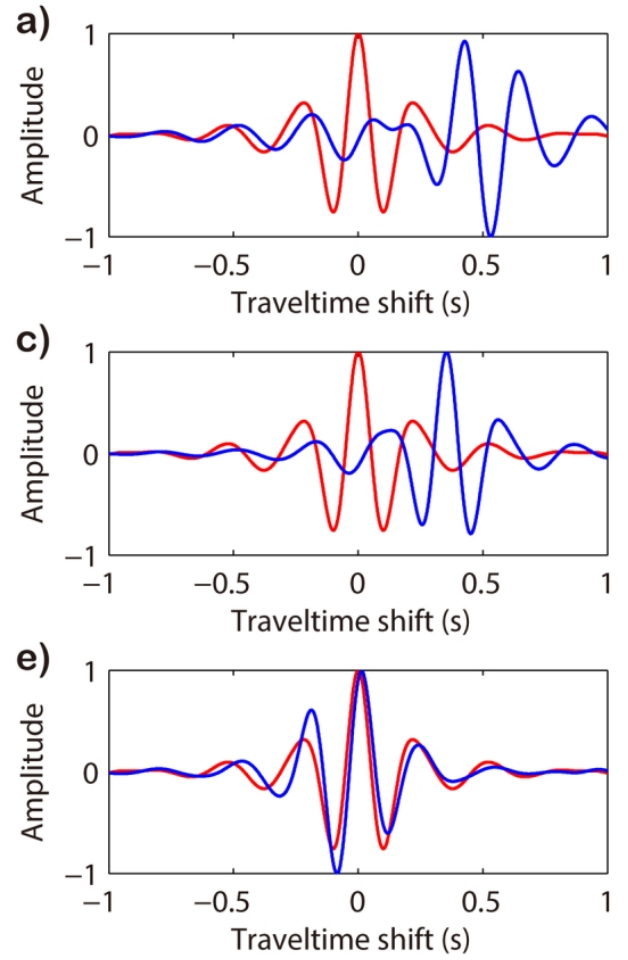
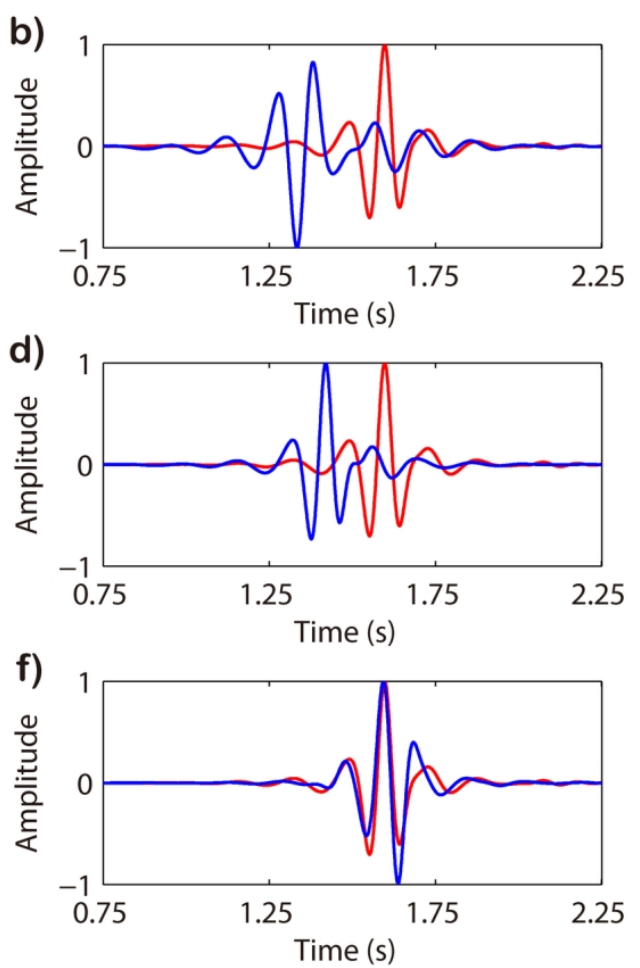

Figure 12. Comparisons of autocorrelation (red) and crosscorrelation (blue), observed (red) and calculated (blue) data for the LCFTI during the iterations: (a) crosscorrelation using the starting model, (b) trace of the starting model, (c) crosscorrelation using the inverted model at the tenth iteration, (d) trace of the inverted model at the tenth iteration, (e) crosscorrelation using the final inverted model, and ( $f$ ) trace of the final LCFTI model.

$145 \times 110 \mathrm{~mm}(300 \times 300$ DPI $)$ 

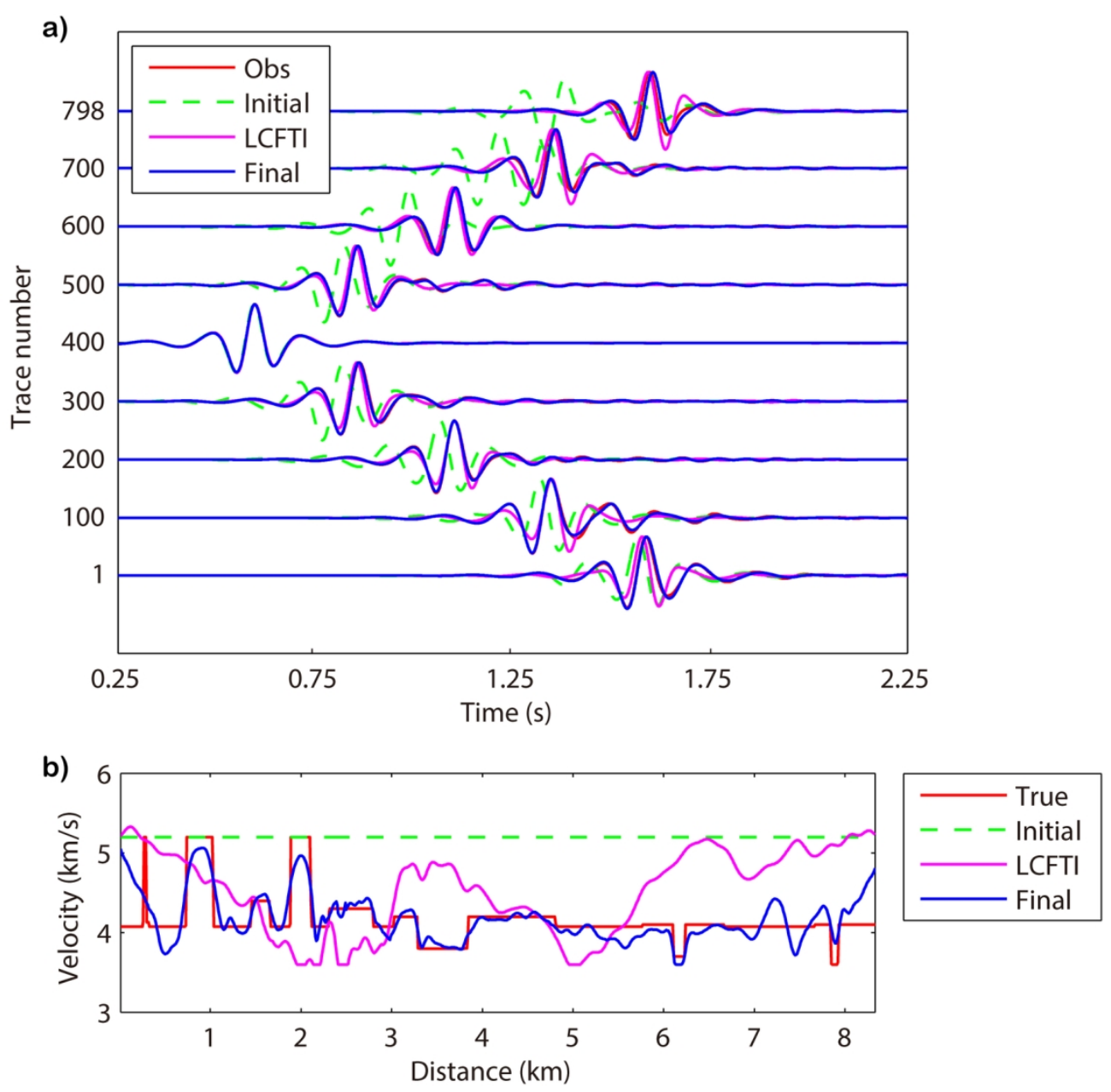

Figure 13. (a) Comparison of traces extracted from the observed, initial, LCFTI, and the final FWI seismic data. (b) Comparison of the true model, initial model, LCFTI result and the final inversion result by combing LCFTI with FWI at depth of $0.6 \mathrm{~km}$.

$139 \times 134 \mathrm{~mm}(300 \times 300 \mathrm{DPI})$ 

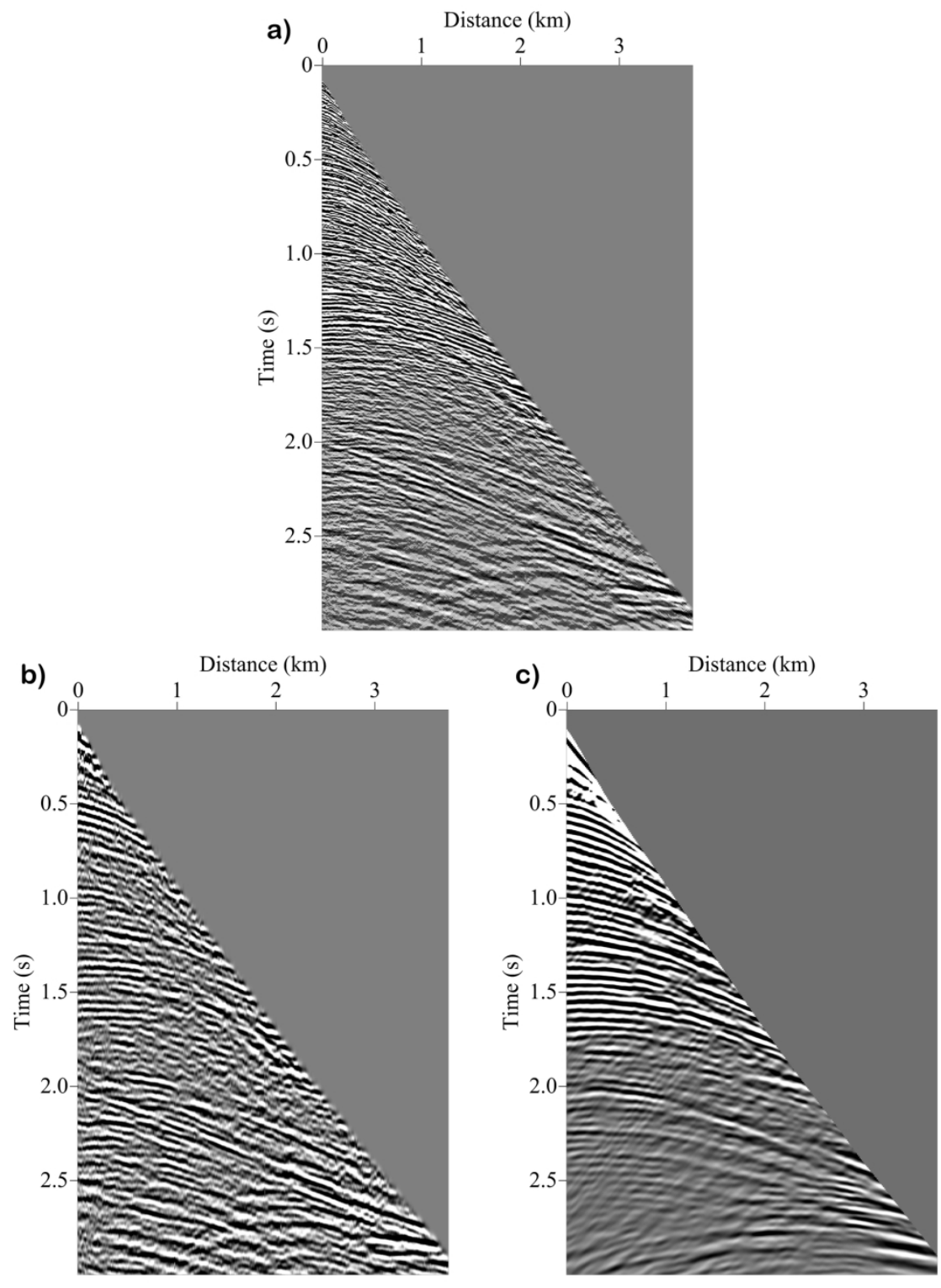

Figure 14. Shot records of (a) the original marine data, (b) the filtered data convolved with the source wavelet, and (c) the synthetic data generated by the final inverted velocity model.

$153 \times 207 \mathrm{~mm}(300 \times 300$ DPI $)$ 

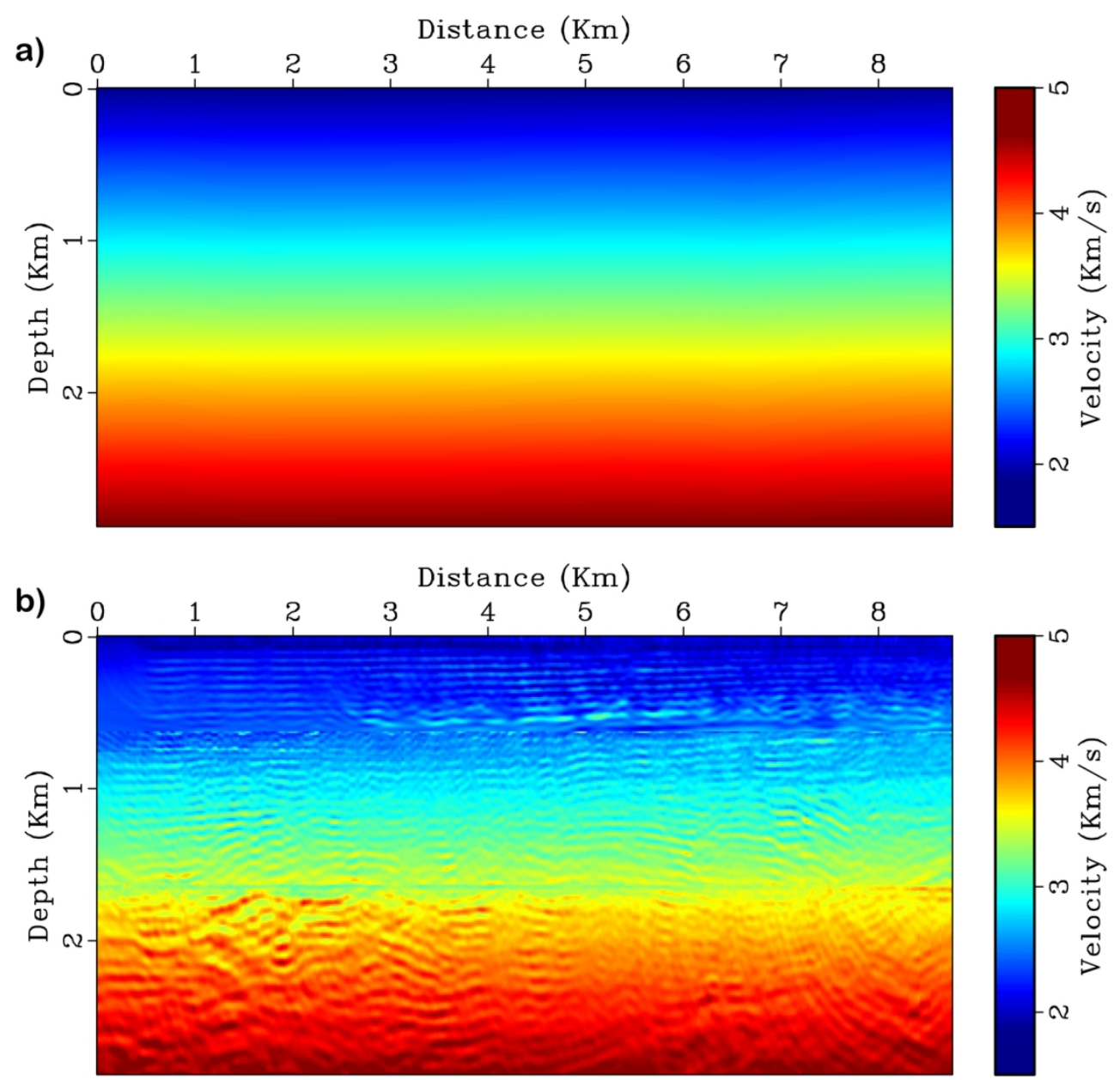

Figure 15. (a) Initial velocity model, and (b) final inverted velocity model by applying LCFTI and traditional FWI.

$145 \times 142 \mathrm{~mm}(300 \times 300$ DPI $)$ 

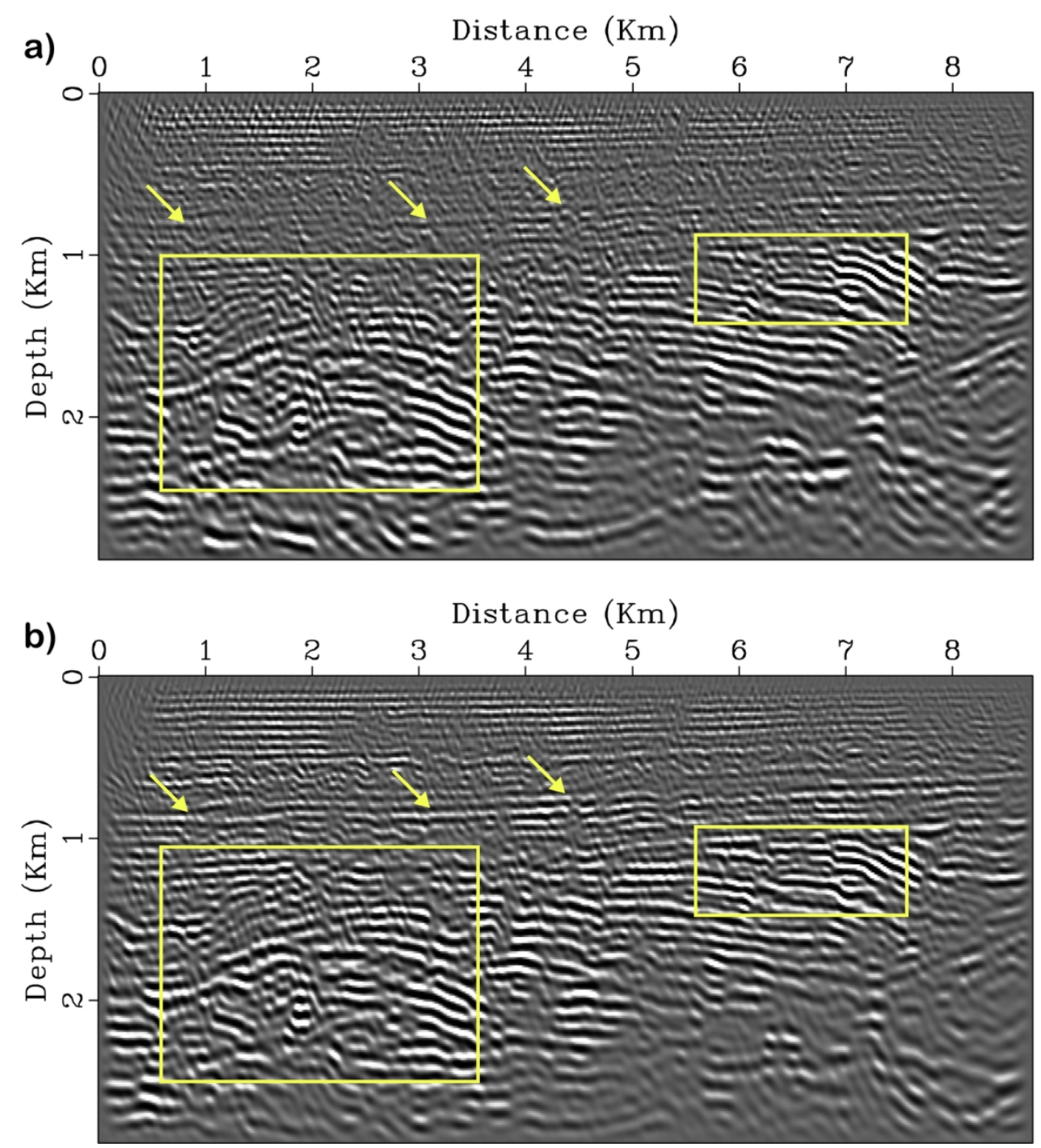

Figure 16. Migration images using (a) the initial velocity and (b) the final inverted velocity model. Yellow arrows and boxes indicate the improvements.

$128 \times 140 \mathrm{~mm}(300 \times 300 \mathrm{DPI})$ 

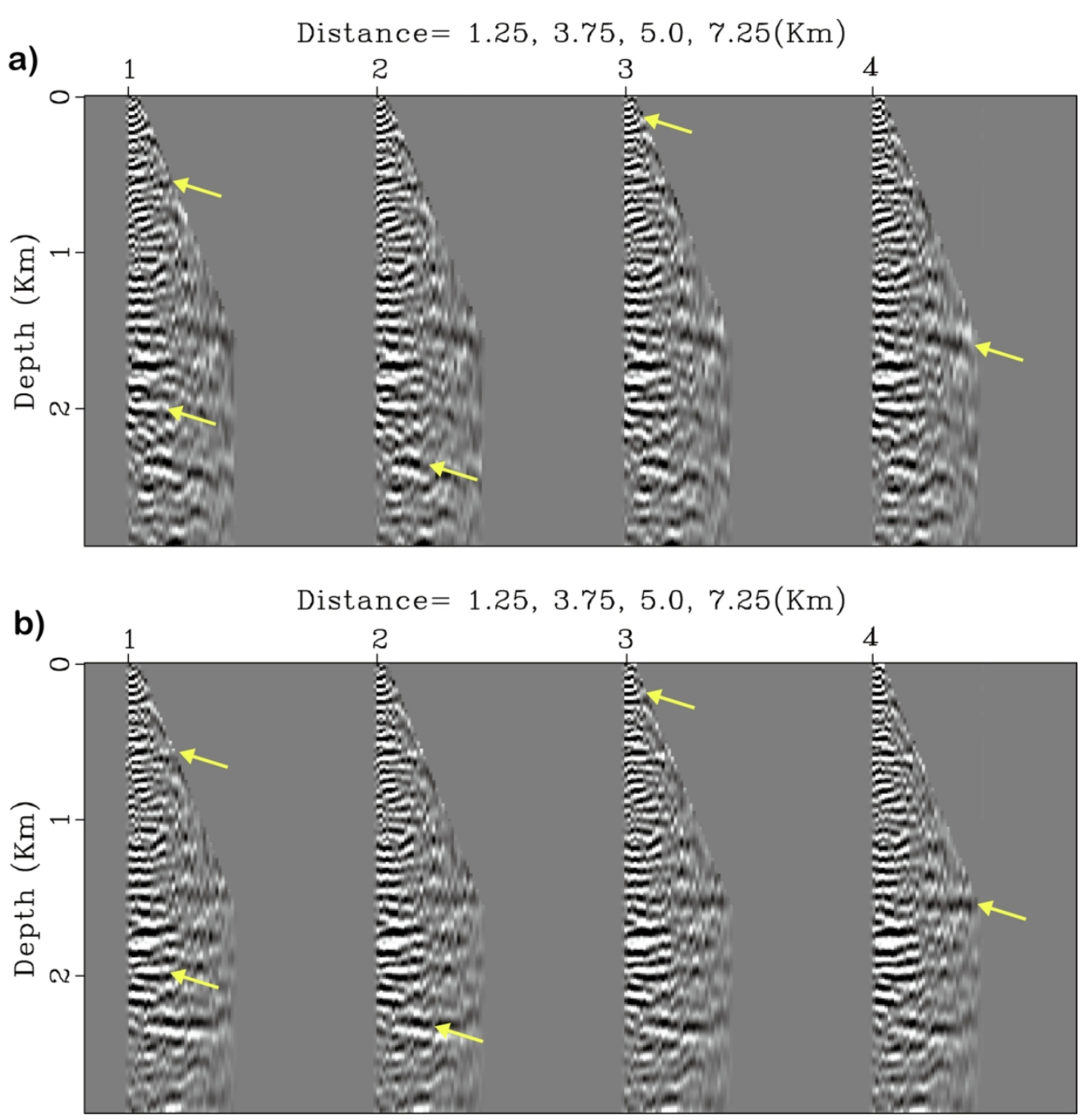

Figure17. Selected CIGs at select spatial locations generated using (a) the initial velocity and (b) the final inverted velocity model. Yellow arrows indicate the improvements.

$135 \times 138 \mathrm{~mm}(300 \times 300 \mathrm{DPI})$ 
DATA AND MATERIALS AVAILABILITY

Data associated with this research are available and can be obtained by contacting the corresponding author. 